\title{
Multiboson effects in multiparticle production
}

\author{
R. Lednicky ${ }^{1,2}$, V. Lyuboshitz ${ }^{1,3}$, K. Mikhailov ${ }^{1,4}$, Yu. Sinyukov ${ }^{1,5}$, \\ A. Stavinsky ${ }^{1,4}$ and B. Erazmus ${ }^{1}$ \\ ${ }^{1}$ SUBATECH, (UMR, Universite, Ecole des Mines, IN2P3/CNRS), \\ 4, rue Alfred Kastler, F-44070 Nantes Cedex 03, France. \\ ${ }^{2}$ Institute of Physics, Na Slovance 2, 18221 Prague 8, Czech Republic. \\ 3 JINR, 141980 Dubna, Moscow region, Russia. \\ ${ }^{4}$ ITEP, B. Cheremushkinskaya 25, 117259 Moscow, Russia. \\ ${ }^{5}$ ITP, Metrologicheskaya 14b, 252143 Kiev, Ukraine.
}

\begin{abstract}
The influence of multiboson effects on pion multiplicities, single-pion spectra and two-pion correlation functions is discussed in terms of an analytically solvable model. The applicability of its basic factorization assumption is clarified. An approximate scaling of the basic observables with the phase space density is demonstrated in the low density (gas) limit. This scaling and also its violation at high densities due to the condensate formation is described by approximate analytical formulae which allow, in principle, for the identification of the multiboson effects among others. For moderate densities indicated by the experimental data, a fast saturation of multiboson effects with the number of contributing cumulants is obtained, allowing for the account of these effects in realistic transport code simulations. At high densities, the spectra are mainly determined by the universal condensate term and the initially narrow Poisson multiplicity distribution approaches a wide Bose-Einstein one. As a result, the intercepts of the inclusive and fixed- $n$ correlation functions (properly normalized to 1 at large relative momenta) approach 2 and 1 , respectively and their widths logarithmically increase with the increasing phase space density. It is shown that the neglect of energy-momentum constraints in the model is justified except near a multipion threshold, where these constraints practically exclude the possibility of a very cold condensate production. It is argued that spectacular multiboson effects are likely to be observed only in the rare events containing sufficiently high density (speckle) fluctuations.
\end{abstract}

25.75.Gz, 05.30.Jp, 24.10.Nz

\section{INTRODUCTION}

In future heavy ion experiments at RHIC and LHC one expects to obtain thousands of pions per a unit rapidity interval. Since the pions are bosons there can be multiboson effects enhancing the production of pions with low relative momenta thus increasing the pion multiplicities, softening their spectra and modifying the correlation functions. Though the present data does not point to any spectacular multiboson effects, one can hope to observe new interesting phenomena like boson condensation or speckles in some rare events or in eventually overpopulated kinematic regions with the pion density in the 6 -dimensional phase space, $f=(2 \pi)^{3} d^{6} n / d^{3} \mathbf{p} d^{3} \mathbf{x}$, of the order of unity (see, e.g., [1][8]).

In the low-density limit $(f \ll 1)$, the mean phase space density at a given momentum $\mathbf{p}$ can be estimated as the mean number of pions interfering with a pion of momentum $\mathbf{p}$ (rapidity $y$ and transverse momentum $\mathbf{p}_{t}$ ) and building the Bose-Einstein (BE) enhancement in the two-pion correlation function [7],8]: $\langle f\rangle_{\mathbf{p}} \sim \pi^{3 / 2} N(\mathbf{p}) / V$, where $N(\mathbf{p})=d^{3} n / d^{3} \mathbf{p}$ and $V=r_{x} r_{y} r_{z}$ is the interference volume defined in terms of the outward $\left(r_{x}\right)$, sideward $\left(r_{y}\right)$ and longitudinal $\left(r_{z}\right)$ interferometry radii. Typically $\langle f\rangle_{\mathbf{p}} \sim 0.1$ for mid-rapidities and $p_{t} \sim\left\langle p_{t}\right\rangle[7]$. The data are also consistent with the phase space density of pions near the local thermal equilibrium [9].

At AGS and SPS energies the interference volume $V$ seems to scale with $d n / d y$ (see, e.g., 10 11) pointing to the freeze-out of the pions at a constant phase space density.] If this trend will survive then there will be no spectacular multiboson effects in the ordinary events at RHIC or even at LHC. In such a situation the standard two-particle interferometry technique could be used to measure the space-time intervals between the production points also in the

\footnotetext{
${ }^{1}$ Similar effect was observed also for protons produced in hadron- and electron-nucleus interactions [12].
} 
future collider experiments. The corresponding interferometry radii for lead-lead collisions at LHC would be however rather large - about $20 \mathrm{fm}$.

The multiboson effects can show up however in certain classes of events. An example is a rapidly expanding system with the entropy much smaller than in the case of total equilibrium. Then a strong transverse flow can lead to rather dense gas of soft pions in the central part of the hydrodynamic tube at the final expansion stage (see, e.g., [13]). Another reason can be the formation of quark-gluon plasma or mixed phase. Due to large gradients of temperature or velocity the hydrodynamic layer near the boundary with vacuum can decay at a large phase space density and lead to pion speckles even at moderate transverse momenta [14].

The dramatic difference in behavior of Boltzman-like gases and dense multiboson systems can lead to serious problems for transport models like RQMD, VENUS, etc., ignoring actually the statistical properties of the particles both in intermediate and final states. In these models the most intensive particle production happens at relatively early evolution (expansion) stage when rather large pion phase space densities can be achieved at RHIC or LHC energies.

Generally, the account of the multiboson effects is extremely difficult task. Even on the neglect of particle interaction in the final state the requirement of the $\mathrm{BE}$ symmetrization leads to severe numerical problems which increase factorially with the number of produced bosons [3, [- In such a situation, it is important that there exists a simple analytically solvable model [5] allowing for a study of the characteristic features of the multiboson systems under various conditions including those near the Bose condensation. In this paper we use this model to demonstrate the influence of the multiboson effects on pion multiplicities, spectra and two-pion correlation functions. Besides the original papers [5]6], similar studies can be found also in 15 17]. Particularly, some of the new aspects of the multiboson effects, like the scaling behavior with the phase space density or the behavior of the (semi-)inclusive correlation functions near the condensation limit were studied in our unpublished paper 15. The present work represents an elaborated version of the latter.

In Section [1] we introduce the space-time description of particle production in terms of Wigner-like densities and discuss their physical meaning and the conditions of their factorization in the model of classical one-particle sources. The multiboson formalism in the factorizable case is set forth in Section III Using this formalism and the simple Gaussian ansatz for the emission function, we present in Section IV the analytical solutions (partly in terms of the recurrence relations) for the multiplicity distribution, single-boson spectra and two-boson correlation functions. In Section $\mathrm{V}$ we compare the results of numerical calculations with the analytical approximations accounting for the approximate scaling behavior in the low density (gas) limit as well as for the condensate formation at high densities. The results are discussed and summarized in Section V1.

\section{SPACE-TIME PICTURE OF PARTICLE PRODUCTION}

\section{A. Wigner-like density}

Let us first consider a process in which, besides others, just $n$ non-identical particles of given types are produced with the 4-momenta $p_{i}=\left\{E_{i}, \mathbf{p}_{i}\right\}$ and Lorentz factors $\gamma_{i}=E_{i} / m_{i}$ (to simplify the notation, we assume that particles are spin-less). The inclusive differential cross section of this process is described by the invariant production amplitude $T_{n}\left(p_{1}, \ldots, p_{n} ; \alpha\right)$ :

$$
\gamma_{1} \cdots \gamma_{n} \frac{d^{3 n} \sigma_{n}}{d^{3} \mathbf{p}_{1} \cdots d^{3} \mathbf{p}_{n}}=\sum_{\alpha}\left|T_{n}\left(p_{1}, \ldots, p_{n} ; \alpha\right)\right|^{2} \equiv \gamma_{1} \cdots \gamma_{n} \sigma_{n} P_{n}\left(\mathbf{p}_{1}, \ldots, \mathbf{p}_{n}\right),
$$

where the sum over the quantum numbers $\alpha$, describing the rest of the produced system, contains also an integration over the momenta of the other produced particles with the energy-momentum conservation taken into account. The non-invariant production probability $P_{n}\left(\mathbf{p}_{1}, \ldots, \mathbf{p}_{n}\right)$ is normalized to unity.

If the particles are identical spin-less bosons, then the production amplitude has to satisfy the requirement of Bose symmetry. Formally, this can be achieved by the substitution of the non-symmetrized amplitude $T_{n}\left(p_{1}, \ldots, p_{n} ; \alpha\right)$ (corresponding to the "switched off" effect of quantum statistics) by a properly symmetrized one:

$$
T_{n}\left(p_{1}, \ldots, p_{n} ; \alpha\right) \rightarrow(n !)^{-1 / 2} \sum_{\sigma} T_{n}\left(p_{\sigma_{1}}, \ldots, p_{\sigma_{n}} ; \alpha\right),
$$

where the sum is over all $n$ ! permutations $\sigma$ of the sequence $\{1,2, \ldots n\}$. 
In the following we will neglect particle interaction in the final state.2 Then the non-symmetrized amplitude $T_{n}\left(p_{1}, \ldots, p_{n} ; \alpha\right) \equiv T_{n}(\mathcal{P} ; \alpha)$ is related to the amplitude in the space-time representation $\mathcal{T}_{n}\left(x_{1}, \ldots, x_{n} ; \alpha\right) \equiv \mathcal{T}_{n}(\mathcal{X} ; \alpha)$ (describing production of the particles in the space-time points with the 4-coordinates $\mathcal{X}=\left\{x_{1}, \ldots, x_{n}\right\}$ ) by the usual Fourier transform:

$$
T_{n}(\mathcal{P} ; \alpha)=\int d \mathcal{X} \mathrm{e}^{i \mathcal{P} \mathcal{X}} \mathcal{T}_{n}(\mathcal{X} ; \alpha)
$$

Inserting (3) into (11) and introducing the space-time density matrix [19]: $\rho_{n}\left(\mathcal{X} ; \mathcal{X}^{\prime}\right)=\sum_{\alpha} \mathcal{T}_{n}(\mathcal{X} ; \alpha) \mathcal{T}_{n}^{*}\left(\mathcal{X}^{\prime} ; \alpha\right)$ and its partial Fourier transform (emission function) - an analogy of the Wigner density [20]:

$$
\widetilde{D}_{n}\left(p_{1}, \bar{x}_{1} ; \ldots ; p_{n}, \bar{x}_{n}\right) \equiv \gamma_{1} \cdots \gamma_{n} \sigma_{n} D_{n}\left(p_{1}, \bar{x}_{1} ; \ldots ; p_{n}, \bar{x}_{n}\right)=\int d \delta \mathrm{e}^{i \mathcal{P} \delta} \rho_{n}\left(\overline{\mathcal{X}}+\frac{1}{2} \delta ; \overline{\mathcal{X}}-\frac{1}{2} \delta\right),
$$

where $\overline{\mathcal{X}}=\frac{1}{2}\left(\mathcal{X}+\mathcal{X}^{\prime}\right), \delta=\mathcal{X}-\mathcal{X}^{\prime}$, we can rewrite the production cross section (1) in the absence of BE effects as:

$$
\frac{d^{3 n} \sigma_{n}}{d^{3} \mathbf{p}_{1} \cdots d^{3} \mathbf{p}_{n}} \equiv \sigma_{n} P_{n}\left(\mathbf{p}_{1}, \ldots, \mathbf{p}_{n}\right)=\sigma_{n} \int d^{4} \bar{x}_{1} \cdots d^{4} \bar{x}_{n} D_{n}\left(p_{1}, \bar{x}_{1} ; \ldots ; p_{n}, \bar{x}_{n}\right) .
$$

Since $D_{n}$ is a real (though not positively defined) function normalized to unity, in accordance with Eq. (5) it can be considered as an approximation to the emission probability of the particles with given 4 -momenta $p_{i}$ in the average space-time points $\bar{x}_{i}=\frac{1}{2}\left(x_{i}+x_{i}^{\prime}\right)$.

The insertion of the symmetrized amplitude (2) into the cross section formula (1) leads to the substitution of the probability $P_{n}$ by a BE modified one $P_{n}^{c}$. For example, in case of a two-boson production process, instead of Eq. (5) we have:

$$
P_{2}^{c}\left(\mathbf{p}_{1}, \mathbf{p}_{2}\right)=\int d^{4} x_{1} d^{4} x_{2}\left[D_{2}\left(p_{1}, x_{1} ; p_{2}, x_{2}\right)+D_{2}\left(p, x_{1} ; p, x_{2}\right) \cos (q x)\right]
$$

where $p=\frac{1}{2}\left(p_{1}+p_{2}\right), q=p_{1}-p_{2}$ and $x=x_{1}-x_{2}$. Clearly, for $n>1$ the probability $P_{n}^{c}$ is no more normalized to unity. The integral over this probability yields the BE weight $\omega_{n}$ of an $n$-boson event produced in the absence of the effect of quantum statistics $\left(\omega_{0}=\omega_{1}=1, \omega_{n}>1\right.$ for $\left.n>1\right)$ :

$$
\omega_{n}=\int d^{3} \mathbf{p}_{1} \cdots d^{3} \mathbf{p}_{n} P_{n}^{c}\left(\mathbf{p}_{1}, \ldots, \mathbf{p}_{n}\right) / \int d^{3} \mathbf{p}_{1} \cdots d^{3} \mathbf{p}_{n} P_{n}\left(\mathbf{p}_{1}, \ldots, \mathbf{p}_{n}\right) .
$$

We will also use the differential BE weights $\omega_{n}^{(k)}\left(\mathbf{p}_{1}, \ldots, \mathbf{p}_{k}\right)$ defined as in Eq. (7) except for a skipped integration over the momenta of particles $1,2, \ldots, k$.

The multiboson problem greatly simplifies (see Section III) in the factorizable case when the $n$-particle emission function and, as a consequence, the non-symmetrized production probability can be written as products of the singleparticle ones:

$$
D_{n}\left(p_{1}, x_{1} ; \ldots ; p_{n}, x_{n}\right)=D\left(p_{1}, x_{1}\right) \cdots D\left(p_{n}, x_{n}\right), \quad P_{n}\left(\mathbf{p}_{1}, \ldots, \mathbf{p}_{n}\right)=P\left(\mathbf{p}_{1}\right) \cdots P\left(\mathbf{p}_{n}\right) .
$$

Consequently, the BE weights are expressed through a universal function $F_{i j}$ (see, e.g., [18]):

$$
\omega_{n}^{(n)}\left(\mathbf{p}_{1}, \mathbf{p}_{2}, \ldots \mathbf{p}_{n}\right)=\sum_{\sigma} \prod_{i=1}^{n} F_{i \sigma_{i}}
$$

where

$$
F_{i j}=\int d^{4} x D\left(p_{i j}, x\right) \cdot \exp \left(i q_{i j} x\right) /\left[P\left(\mathbf{p}_{i}\right) P\left(\mathbf{p}_{j}\right)\right]^{1 / 2},
$$

$p_{i j}=\frac{1}{2}\left(p_{i}+p_{j}\right)$ and $q_{i j}=p_{i}-p_{j}$. The sum in Eq. (9) is over $n$ ! possible permutations $\sigma$ of the sequence $\{1,2, \ldots n\}$. For example, in the two-boson case, we have

$$
\omega_{2}^{(2)}\left(\mathbf{p}_{1}, \mathbf{p}_{2}\right)=F_{11} F_{22}+F_{12} F_{21} \equiv 1+K_{2}^{(2)}\left(\mathbf{p}_{1}, \mathbf{p}_{2}\right),
$$

where $K_{2}^{(2)}=F_{12} F_{21}$ is so called differential cumulant (see Section III).

\footnotetext{
${ }^{2}$ This is more or less valid assumption for neutral pions but not for the charged ones. For the treatment of multiboson effects in the case of interacting pions see, e.g., [18].
} 


\section{B. One-particle sources}

To clarify the physical meaning of the emission function and the factorization assumption (8), let us follow Kopylov and Podgoretsky (see, e.g., 正) and assume that particles $1,2, \ldots$ are emitted by one-particle sources $A, B, \ldots$ which are considered as classical so they can be treated by parameters and not by amplitudes. Thus the 4-coordinates of the source centers $x_{A}, x_{B}, \ldots$ and other source characteristics in the model can be considered as a part of the quantum numbers $\alpha \equiv\left\{x_{A}, x_{B}, \ldots \alpha^{\prime}\right\}$. It was pointed out by Kopylov and Podgoretsky that the BE effect is mainly determined by the phase factor $\exp \left(i p_{1} x_{A}+i p_{2} x_{B}+\cdots\right)$ contained in the amplitude $T_{n}\left(p_{1}, \ldots, p_{n} ; \alpha\right)$.

Let us first consider the production of only one boson. Assuming the translation invariance of the decay amplitudes $\widetilde{u}$, we can write the single-boson amplitude in the 4-coordinate representation as:

$$
\mathcal{T}\left(x_{1} ; \alpha\right) \equiv \widetilde{t}\left(x_{1}-x_{A} ; \alpha\right)=\widetilde{u}\left(x_{1}-x_{A} ; \alpha^{\prime}\right) v(\alpha),
$$

where $\alpha=\left\{x_{A}, \alpha^{\prime}\right\}$ and $\alpha^{\prime}=\{A, \ldots\}$. Inserting (12) into (3) and introducing the Fourier transform

$$
t(p ; \alpha)=\int d^{4} \xi \mathrm{e}^{i p \xi} \tilde{t}(\xi ; \alpha) \equiv u\left(p ; \alpha^{\prime}\right) v(\alpha),
$$

we obtain the Kopylov-Podgoretsky ansatz:

$$
T(p ; \alpha)=\mathrm{e}^{i p x_{A}} t(p ; \alpha) \equiv \mathrm{e}^{i p x_{A}} u\left(p ; \alpha^{\prime}\right) v(\alpha) .
$$

For the production probability we have

$$
P^{c}(\mathbf{p})=P(\mathbf{p}) \equiv \int d^{4} x_{A} W\left(p, x_{A}\right)=\int d^{4} x_{A} \sum_{\alpha^{\prime}}\left|t\left(p ;\left\{x_{A}, \alpha^{\prime}\right\}\right)\right|^{2},
$$

where we have introduced the (true) emission probability $W\left(\mathbf{p}, x_{A}\right)$. Similarly, if two bosons are produced, the production probability takes on the form:

$$
P_{2}^{c}\left(\mathbf{p}_{1}, \mathbf{p}_{2}\right)=\int d^{4} x_{A} d^{4} x_{B} \sum_{\alpha^{\prime}}\left\{\left|t\left(p_{1}, p_{2} ; \alpha\right)\right|^{2}+R e\left[t\left(p_{1}, p_{2} ; \alpha\right) t^{*}\left(p_{2}, p_{1} ; \alpha\right) \mathrm{e}^{i q x}\right]\right\} .
$$

Note that here $x=x_{A}-x_{B}$ and, as usual, $q=p_{1}-p_{2}$.

The emission function $D$ can be expressed through the Kopylov-Podgoretsky amplitudes $t(p ; \alpha)$ continued off massshell. Using the inverted Fourier transform in Eq. (13), we get from Eq. (4) [18]:

$$
\begin{gathered}
D\left(p, \bar{x}_{1}\right)=\frac{1}{(2 \pi)^{4}} \sum_{\alpha^{\prime}} \int d^{4} x_{A} d^{4} \kappa \mathrm{e}^{i \kappa\left(x_{A}-\bar{x}_{1}\right)} \cdot t\left(p+\frac{1}{2} \kappa ;\left\{x_{A}, \alpha^{\prime}\right\}\right) t^{*}\left(p-\frac{1}{2} \kappa ;\left\{x_{A}, \alpha^{\prime}\right\}\right) \\
=\frac{1}{(2 \pi)^{4}} \sum_{\alpha^{\prime}} \int d^{4} x_{A} d^{4} \kappa \mathrm{e}^{i \kappa\left(x_{A}-\bar{x}_{1}\right)} \cdot u\left(p+\frac{1}{2} \kappa ; \alpha^{\prime}\right) u^{*}\left(p-\frac{1}{2} \kappa ; \alpha^{\prime}\right)|v(\alpha)|^{2} .
\end{gathered}
$$

It is clear from Eq. (13) that the momentum dependence of the amplitude $t(p ; \alpha)$ is determined by the spacetime extent of the one-particle source. For example, assuming that the source emits a particle independently of the quantum numbers $\alpha^{\prime}$ except for the source type $A: \widetilde{u}\left(\xi ; \alpha^{\prime}\right)=\widetilde{u}(\xi ; A)$ and that the distribution of the emission points in the source rest frame is given by a simple Gaussian with the width parameters $r_{A}$ and $\tau_{A}$ characterizing the proper space-time sizes of the source $A$, we obtain in case of a source at rest: ${ }^{3}$

$$
t(p ; \alpha) \sim \exp \left(-\frac{1}{2} r_{A}^{2} \mathbf{p}^{2}-\frac{1}{2} \tau_{A}^{2} p_{0}^{2}\right) v(\alpha) .
$$

The probability $|v(\alpha)|^{2}$ describes the production of particle sources and depends on the 4-coordinates $x_{A}$ of the source centers. In the following we will take it also in a simple Gaussian form with the width parameters $\widetilde{r}_{0}$ and $\widetilde{\tau}_{0}$ characterizing the space-time extent of the source production region.

\footnotetext{
${ }^{3}$ Note that Eq. (18) is valid also off mass-shell when $p_{0} \neq E$. For a source moving with a non-relativistic velocity $\boldsymbol{\beta}_{A}$, the substitution $\mathbf{p} \rightarrow \mathbf{p}-\mathbf{p}_{A}$ has to be done in Eq. (18), where $\mathbf{p}_{A}=m \boldsymbol{\beta}_{A}$ is a mean 3-momentum of the particle emitted by a source $A$.
} 
Comparing Eqs. (15) and (17), it can be seen that the emission function $D$ is more spread in space and time than the emission probability $W$. In particular, the Gaussian parameterizations of $\widetilde{u}(\xi ; A)$ and $|v(\alpha)|^{2}$ yield:

$$
W(p, x) \sim \exp \left(-r_{A}^{2} \mathbf{p}^{2}-\tau_{A}^{2} p_{0}^{2}\right) \exp \left(-\frac{\mathbf{x}^{2}}{2 \widetilde{r}_{0}^{2}}-\frac{x_{0}^{2}}{2 \widetilde{\tau}_{0}^{2}}\right)
$$

and

$$
D(p, x) \sim \exp \left(-r_{A}^{2} \mathbf{p}^{2}-\tau_{A}^{2} p_{0}^{2}\right) \exp \left(-\frac{\mathbf{x}^{2}}{2 \widetilde{r}_{0}^{2}+r_{A}^{2}}-\frac{x_{0}^{2}}{2 \widetilde{\tau}_{0}^{2}+\tau_{A}^{2}}\right) .
$$

Clearly, the factorized form (8) for the emission function is recovered in case of independent sources (i.e. sources having no quantum numbers in common) assuming a unique mechanism of their production. Generally, the latter condition may not be fulfilled, e.g. in case of heavy ion collisions without selection of the impact parameter. Then, even for independent sources, Eq. (8) will be substituted by a weighted sum of factorized terms corresponding to different single-particle emission functions.

The single-particle density generally contains contributions from the sources of different type (e.g. different resonances). It is interesting to note that in case of only one source type (i.e. universal source parameters) and on condition of sufficiently slow relative motion of the sources contributing to low- $|q|$ pairs (e.g., due to limited source decay momentum), the BE correlation effect in the two-boson case is solely determined by the characteristic space-time distance between the source centers:

$$
P_{2}^{c}\left(\mathbf{p}_{1}, \mathbf{p}_{2}\right)=P\left(\mathbf{p}_{1}\right) P\left(\mathbf{p}_{2}\right)\left[1+\exp \left(-\widetilde{r}_{0}^{2} \mathbf{q}^{2}-\widetilde{\tau}_{0}^{2} q_{0}^{2}\right)\right] .
$$

This result follows from Eq. (6) or, in terms of the Kopylov-Podgoretsky emission amplitudes, immediately from Eq. (16). If the sources of interfering bosons move with non-relativistic velocities $\boldsymbol{\beta}_{A} \equiv \mathbf{p}_{A} / m$ and the distribution of their characteristics is given by a product of $\exp \left(-\mathbf{p}_{A}^{2} / 2 \Delta_{0}^{2}\right)$ and the same Gaussian as before (i.e. the residual slow relative motion decouples from other source characteristics), we still arrive at Eqs. (19), (20), up to a substitution $r_{A}^{2} \mathbf{p}^{2} \rightarrow r_{A}^{2} \mathbf{p}^{2} /\left[2\left(r_{A} \Delta_{0}\right)^{2}+1\right]$ corresponding to a widening of the momentum distribution due to the source relative motion. Note that the factorized form of the multiparticle density is not destroyed by this motion. The latter however influences the two-boson correlation function which now becomes sensitive to the source size $r_{A}$ even in case of one source type, Eq. (21) being modified by the substitution $\widetilde{r}_{0}^{2} \rightarrow \widetilde{r}_{0}^{2}+r_{A}^{2} /\left[2+\left(r_{A} \Delta_{0}\right)^{-2}\right]$.

The space-time extent of one-particle sources can be usually considered much smaller than the characteristic spacetime distance between their centers $\left(r_{A} \ll \widetilde{r}_{0}, \tau_{A} \ll \widetilde{\tau}_{0}\right)$. The 4-momentum dependence of one-particle amplitudes is then negligible when varying the particle 4 -momenta by the amount $\sim \widetilde{r}_{0}^{-1}, \widetilde{\tau}_{0}^{-1}$ characteristic for the interference effect. On such a smoothness condition, there is practically no difference between the emission function $D$ and the emission probability $W$ and both Eqs. (6) and (16) yield the well known result of Kopylov-Podgoretsky for the production probability of two identical bosons:

$$
P_{2}^{c}\left(\mathbf{p}_{1}, \mathbf{p}_{2}\right) \doteq P\left(\mathbf{p}_{1}, \mathbf{p}_{2}\right)\left[1+\left\langle\cos \left(q\left(x_{1}-x_{2}\right)\right)\right\rangle\right] .
$$

\section{MULTIBOSON FORMALISM IN FACTORIZABLE CASE}

The multiboson effects can be practically treated provided that we can neglect particle interaction in the final state and assume independent emission of non-interfering particles (a valid assumption for heavy ion collisions), supplemented by the requirement of a universal single-particle emission function $D(p, x)$ for the detected class of events. We can then use Eq. (8) expressing the $n$-particle emission function as a product of the single-particle ones. Then, similar to refs. [5,6] it is convenient to define the functions

$$
\begin{aligned}
G_{1}\left(\mathbf{p}_{1}, \mathbf{p}_{2}\right) & =\int d^{4} x D\left(\frac{1}{2}\left(p_{1}+p_{2}\right), x\right) \cdot \exp \left(i\left(p_{1}-p_{2}\right) x\right), \\
G_{n}\left(\mathbf{p}_{1}, \mathbf{p}_{2}\right) & =\int d^{3} \mathbf{k}_{2} \ldots d^{3} \mathbf{k}_{n} G_{1}\left(\mathbf{p}_{1}, \mathbf{k}_{2}\right) \ldots G_{1}\left(\mathbf{k}_{n}, \mathbf{p}_{2}\right) \\
& \equiv \int d^{3} \mathbf{k}_{2} G_{n-1}\left(\mathbf{p}_{1}, \mathbf{k}_{2}\right) G_{1}\left(\mathbf{k}_{2}, \mathbf{p}_{2}\right), \\
g_{n} & =\int d^{3} \mathbf{p} G_{n}(\mathbf{p}, \mathbf{p}) .
\end{aligned}
$$


The function $G_{1}$ at equal momenta is just the initial (not affected by the multiboson effects) single-boson spectrum normalized to unity:

$$
P(\mathbf{p})=G_{1}(\mathbf{p}, \mathbf{p}), \quad \int d^{3} \mathbf{p} P(\mathbf{p}) \equiv g_{1}=1 .
$$

The related quantities are so called cumulants

$$
\begin{aligned}
K_{n}^{(2)}\left(\mathbf{p}_{1}, \mathbf{p}_{2}\right) & =(n-2) ! \sum_{i=1}^{n-1} G_{i}\left(\mathbf{p}_{1}, \mathbf{p}_{2}\right) G_{n-i}\left(\mathbf{p}_{2}, \mathbf{p}_{1}\right) /\left[P\left(\mathbf{p}_{1}\right) P\left(\mathbf{p}_{2}\right)\right], \\
K_{n}^{(1)}(\mathbf{p}) & =(n-1) ! G_{n}(\mathbf{p}, \mathbf{p}) / P(\mathbf{p}) \\
K_{n} & =(n-1) ! g_{n} .
\end{aligned}
$$

It can be shown that the $\mathrm{BE}$ weight of an event with $\mathrm{n}$ identical spin-zero bosons is determined through the cumulants $K_{j}$ by the recurrence relation [18]:

$$
\omega_{n}=C_{0}^{n-1} K_{1} \omega_{n-1}+C_{1}^{n-1} K_{2} \omega_{n-2}+\ldots+C_{n-1}^{n-1} K_{n} \omega_{0}
$$

with $\omega_{0}=\omega_{1}=1 ; C_{i}^{n-1}=(n-1) ! /[i !(n-1-i) !]$ are the usual combinatorial numbers. For example, $\omega_{2}=1+K_{2}$ and $\omega_{3}=1+3 K_{2}+K_{3}$. One can check that $\omega_{n}=n$ ! provided that all the elementary one-particle sources are situated at one and the same space-time point so that all the single-boson states are identical and $K_{j+1}=j !$. In the other extreme case of a large phase space volume and $n^{2} K_{2} \ll 1$, we can neglect the contribution of the higher order cumulants except for the first power of $K_{2}$ and write:

$$
\omega_{n} \doteq 1+C_{2}^{n} K_{2}
$$

Given the initial multiplicity distribution $\widetilde{w}(n)$, the BE affected one is easily calculated using the BE weights $\omega_{n}$ :

$$
w(n)=\omega_{n} \widetilde{w}(n) / \sum_{j=0}^{\infty} \omega_{j} \widetilde{w}(j) .
$$

Particularly, assuming the initial Poissonian distribution with the mean multiplicity $\eta: \widetilde{w}(n)=\mathrm{e}^{-\eta} \eta^{n} / n$ !, we get:

$$
w(n)=\omega_{n} \frac{\eta^{n}}{n !} / \sum_{j=0}^{\infty} \omega_{j} \frac{\eta^{j}}{j !} .
$$

Similarly, the BE affected single- and two-boson spectra, respectively normalized to $n$ and $n(n-1)$, can be written as

$$
N_{n}^{(1)}(\mathbf{p}) \equiv N_{n}(\mathbf{p})=n \omega_{n}^{(1)}(\mathbf{p}) \cdot P(\mathbf{p}) / \omega_{n}
$$

and

$$
N_{n}^{(2)}\left(\mathbf{p}_{1}, \mathbf{p}_{2}\right)=n(n-1) \omega_{n}^{(2)}\left(\mathbf{p}_{1}, \mathbf{p}_{2}\right) P\left(\mathbf{p}_{1}\right) P\left(\mathbf{p}_{2}\right) / \omega_{n}
$$

where the differential BE weights $\omega_{n}^{(1)}(\mathbf{p})$ and $\omega_{n}^{(2)}\left(\mathbf{p}_{1}, \mathbf{p}_{2}\right)$ are expressed through the differential cumulants $K_{n}^{(1)}(\mathbf{p})$ and $K_{n}^{(2)}\left(\mathbf{p}_{1}, \mathbf{p}_{2}\right)$ 15]:

$$
\begin{array}{r}
\omega_{n}^{(1)}(\mathbf{p}) \equiv \int d^{3} \mathbf{p}^{\prime} \omega_{n}^{(2)}\left(\mathbf{p}, \mathbf{p}^{\prime}\right) P\left(\mathbf{p}^{\prime}\right)=\sum_{j=0}^{n-1} C_{j}^{n-1} K_{j+1}^{(1)}(\mathbf{p}) \omega_{n-1-j} \\
\omega_{n}^{(2)}\left(\mathbf{p}_{1}, \mathbf{p}_{2}\right)=\sum_{j=0}^{n-2} C_{j}^{n-2} \omega_{n-2-j}\left[\sum_{l=0}^{j} C_{l}^{j} K_{l+1}^{(1)}\left(\mathbf{p}_{1}\right) K_{j-l+1}^{(1)}\left(\mathbf{p}_{2}\right)+K_{j+2}^{(2)}\left(\mathbf{p}_{1}, \mathbf{p}_{2}\right)\right] .
\end{array}
$$

\footnotetext{
${ }^{4}$ This situation is similar (flat correlation function) though different from the case of the emission of so called coherent bosons for which there is no enhancement factor. In fact, when the one-particle sources become closer and closer, so that their distances are less than the wave length of the emitted bosons, they can no more be considered as independent ones and a multiparticle source of non-interfering bosons has to be introduced [21]. To quantify the transition to the non-interfering bosons a concept of the coherence length can be used [22].
} 
The differential weight $\omega_{n}^{(2)}\left(\mathbf{p}_{1}, \mathbf{p}_{2}\right)$ can be considered as a two-particle correlation function measuring the BE effect on the initial uncorrelated two-particle spectrum $\widetilde{N}_{n}^{(2)}\left(\mathbf{p}_{1}, \mathbf{p}_{2}\right)=n(n-1) P\left(\mathbf{p}_{1}\right) P\left(\mathbf{p}_{2}\right)$, with the normalization

$$
\int d^{3} \mathbf{p}_{1} d^{3} \mathbf{p}_{2} \omega_{n}^{(2)}\left(\mathbf{p}_{1}, \mathbf{p}_{2}\right) P\left(\mathbf{p}_{1}\right) P\left(\mathbf{p}_{2}\right)=\omega_{n}
$$

Usually the correlation function is normalized to unity at a large $|\mathbf{q}|$. Such a normalization is approximately satisfied for the correlation function defined as:

$$
R_{n}\left(\mathbf{p}_{1}, \mathbf{p}_{2}\right)=N_{n}^{(2)}\left(\mathbf{p}_{1}, \mathbf{p}_{2}\right) / \widetilde{N}_{n}^{(2)}\left(\mathbf{p}_{1}, \mathbf{p}_{2}\right) \equiv \omega_{n}^{(2)}\left(\mathbf{p}_{1}, \mathbf{p}_{2}\right) / \omega_{n}
$$

In practice, the two-particle correlation function is defined through the observable spectra as:

$$
R_{n}\left(\mathbf{p}_{1}, \mathbf{p}_{2}\right)=c_{n} N_{n}^{(2)}\left(\mathbf{p}_{1}, \mathbf{p}_{2}\right) /\left[N_{n}^{(1)}\left(\mathbf{p}_{1}\right) N_{n}^{(1)}\left(\mathbf{p}_{2}\right)\right] .
$$

Similarly, the (semi-)inclusive correlation function is defined as

$$
R\left(\mathbf{p}_{1}, \mathbf{p}_{2}\right)=c N^{(2)}\left(\mathbf{p}_{1}, \mathbf{p}_{2}\right) /\left[N^{(1)}\left(\mathbf{p}_{1}\right) N^{(1)}\left(\mathbf{p}_{2}\right)\right],
$$

where

$$
N^{(1)}(\mathbf{p})=\sum_{n} w(n) N_{n}^{(1)}(\mathbf{p}), \quad N^{(2)}\left(\mathbf{p}_{1}, \mathbf{p}_{2}\right)=\sum_{n} w(n) N_{n}^{(2)}\left(\mathbf{p}_{1}, \mathbf{p}_{2}\right)
$$

are the corresponding (semi-)inclusive single- and two-particle spectra, $w(n)$ is the normalized multiplicity distribution accounting for the BE effect according to Eq. (28). Later on, using an analytical Gaussian model for the emission function, we show that the normalization constant $c_{n}$ can be expressed through the BE weights as:

$$
c_{n}=n \omega_{n-1}^{2} /\left[(n-1) \omega_{n} \omega_{n-2}\right]
$$

and that $c=1$ for the inclusive correlation function provided a Poissonian multiplicity distribution of the initially uncorrelated bosons.

As one can see from formulae (30)-(32), the multiboson correlations lead to distortions of the initial single- and two-particle distributions. Such distortions are small in the case of interference of only two or three identical particles. However, they can become essential for the events with a large number of identical bosons due to factorially increasing number of the correction terms [3] (see also [1] and [5]). For the processes characterized by a high $(>0.1)$ phase space density of the identical bosons at the freeze-out time the multiboson effects can no more be considered as a correction [3].

To account for the multiboson symmetrization effect in the event simulators, a phase space weighting procedure was used with weights in the form of a normalized square of the sum of $n$ ! plane waves [3. appears not practical for a large $n$ due to the factorially large number of the terms to be computed to calculate the weight and, due to large weight fluctuations. These fluctuations can be substantially reduced by weighting only in the momentum space. The corresponding BE weights are given in Eq. (9). They are expressed through the universal function (10) which is simply related with the function $G_{1}$ :

$$
F_{i j}=G_{1}\left(\mathbf{p}_{i}, \mathbf{p}_{j}\right) /\left[P\left(\mathbf{p}_{i}\right) P\left(\mathbf{p}_{j}\right)\right]^{1 / 2} .
$$

On the condition of sufficient smoothness of the single-particle spectra, we can put

$$
F_{i j} \doteq \int d^{4} x D\left(p_{i j}, x\right) \cdot \exp \left(i q_{i j} x\right) / \int d^{4} x D\left(p_{i j}, x\right)
$$

where $p_{i j}=\frac{1}{2}\left(p_{i}+p_{j}\right)$ and $q_{i j}=p_{i}-p_{j}$. This function can then be calculated as suggested in [18]:

$$
F_{i j}=\left\langle\exp \left(i q_{i j} x_{k}\right)\right\rangle_{\mathbf{p}_{i j}},
$$

where the averaging is done over all simulated phase space points $\left\{p_{k}, x_{k}\right\}$ such that $\mathbf{p}_{k}$ is close to a given 3 -momentum $\mathbf{p}_{i j}$. However, there is still the problem with factorially large number of the terms required to calculate the weight according to Eq. (9). 
Fortunately, when calculating only single- or two-particle distributions according to Eqs. (30) or (31), this number is strongly reduced (eaten by the combinatorial numbers $C_{j}^{m}$ in Eqs. (32)). We should however perform integration over momenta of one or more particles to determine the integrated cumulants $K_{n}^{(2)}\left(\mathbf{p}_{1}, \mathbf{p}_{2}\right), K_{n}^{(1)}(\mathbf{p})$ and $K_{n}$.

The numerical averaging of the cumulants of all orders is a difficult task. In case of large multiplicities of identical bosons $(n>20)$ this is practically possible in the models with a symmetric emission function (allowing to use a special Monte Carlo technique) [3] or with a simple analytical parameterization of this function [5]6]. For example, in ref. [6] the corrections to multiplicity distributions, single-particle spectra and two-particle correlation functions were calculated using the relativistic Bjorken model [23] for the emission function. To compute cumulants up to tenth order, the integration was performed analytically over the space-time coordinates and numerically over the momenta.

Generally, for realistic models used to predict particle production in ultra-relativistic heavy-ion collisions, the numerical limitations allow to determine only a few lowest order cumulants (up to about the fourth order) [18]. Fortunately, since the interferometry measurements point out to a moderate pion freeze-out phase space density of $\sim 0.1$, the lowest order cumulant approximation appears to be reasonable for typical events in present and likely also in future heavy-ion experiments (see Section $\mathrm{V}$ ). At the same time, even in the absence of strong multiboson effects, their account can still be important for realistic simulations of heavy ion collisions [18].

\section{ANALYTICAL MODEL}

To study the multiboson effects in a dense pion gas, we use a simple model assuming independent particle emission (see Eq. (8)) with the Gaussian ansatz for the single-boson emission function $D(p, x)$ [5]:

$$
D(p, x)=\frac{1}{\left(2 \pi r_{0} \Delta\right)^{3}} \exp \left(-\frac{\mathbf{p}^{2}}{2 \Delta^{2}}-\frac{\mathbf{r}^{2}}{2 r_{0}^{2}}\right) \delta(t) .
$$

Note that this ansatz corresponds to the independent one-particle sources of Kopylov and Podgoretsky, all of the same type, characterized by a universal size of $\sim(2 \Delta)^{-1}$, with the centers distributed according to a Gaussian of a dispersion $\widetilde{r}_{0}^{2}=r_{0}^{2}-(2 \Delta)^{-2}$ (see Eq. (20)). Then, in the low density limit but regardless of the validity of the smoothness condition $\widetilde{r}_{0} \gg(2 \Delta)^{-1}$ (see, however, the footnote after Eq. (26) concerning the independence assumption), the correlation function of two non-interacting identical particles measures the dispersion of the relative 4 -coordinates $\widetilde{x}$ of the centers of the one-particle sources as the inverse width squared of the correlation effect seen in the relative momenta $\mathbf{q}=\mathbf{p}_{1}-\mathbf{p}_{2}$ [1]. For spin-0 bosons

$$
R\left(\mathbf{p}_{1}, \mathbf{p}_{2}\right)=1+\langle\cos (q \widetilde{x})\rangle=1+\exp \left(-\widetilde{r}_{0}^{2} \mathbf{q}^{2}\right) .
$$

In this model the initial boson phase space density (not affected by the BE effect) is given by

$$
\tilde{f}_{n}(\mathbf{p}, \mathbf{x})=\frac{n}{\left(r_{0} \Delta\right)^{3}} \exp \left(-\frac{\mathbf{p}^{2}}{2 \Delta^{2}}-\frac{\mathbf{x}^{2}}{2 r_{0}^{2}}\right) .
$$

The mean densities at a fixed boson momentum $\mathbf{p}$ and averaged over all phase space are

$$
\left\langle\tilde{f}_{n}\right\rangle_{\mathbf{p}} \equiv \int d^{3} \mathbf{x}\left(\tilde{f}_{n}\right)^{2} / \int d^{3} \mathbf{x} \tilde{f}_{n}=\frac{n}{\left(\sqrt{2} r_{0} \Delta\right)^{3}} \exp \left(-\frac{\mathbf{p}^{2}}{2 \Delta^{2}}\right)
$$

and

$$
\left\langle\tilde{f}_{n}\right\rangle \equiv \int d^{3} \mathbf{x} d^{3} \mathbf{p}\left(\tilde{f}_{n}\right)^{2} / \int d^{3} \mathbf{x} d^{3} \mathbf{p} \tilde{f}_{n}=n /\left(2 r_{0} \Delta\right)^{3},
$$

respectively. Similarly, the initial inclusive densities $\tilde{f}(\mathbf{p}, \mathbf{x}),\langle\tilde{f}\rangle_{\mathbf{p}}$ and $\langle\tilde{f}\rangle$ are given by Eqs. (44)-(46) with the multiplicity $n$ substituted by the initial mean multiplicity.

It is worth noting an approximate equality (see also a model independent prove in [7]) between the mean phase space density in the low density limit

$$
\langle\tilde{f}\rangle_{\mathbf{p}}=\frac{\eta}{\left(\sqrt{2} r_{0} \Delta\right)^{3}} \exp \left(-\frac{\mathbf{p}^{2}}{2 \Delta^{2}}\right) \doteq \frac{\pi^{3 / 2}}{r_{0}^{3}} N(\mathbf{p})
$$

and the mean number of pions building the BE enhancement in the two-pion correlation function 


$$
\int d^{3} \mathbf{q}\left[R\left(p_{1}, p_{2}\right)-1\right] N\left(\mathbf{p}_{1}\right) N\left(\mathbf{p}_{2}\right) / N(\mathbf{p})=\frac{\pi^{3 / 2}}{\widetilde{r}_{0}^{3}} N(\mathbf{p}) \approx\langle\tilde{f}\rangle_{\mathbf{p}}
$$

$\mathbf{p}_{1,2}=\mathbf{p} \pm \mathbf{q} / 2$. This equality is valid up to relative corrections $O\left(\left(r_{0} \Delta\right)^{-2}\right)$ and $O\left(\langle\tilde{f}\rangle_{\mathbf{p}}\right)$, the latter representing an impact of the BE correlations on the single-boson spectrum (see Section VB).

It is important that the Gaussian ansatz in Eq. (42) allows to express the functions $G_{n}\left(\mathbf{p}_{1}, \mathbf{p}_{2}\right)$ and the integrals $g_{n}$ (see Eqs. 231) in simple analytical forms [15]:

$$
\begin{aligned}
G_{n}\left(\mathbf{p}_{1}, \mathbf{p}_{2}\right) & =\left(2 \pi \Delta^{2} A_{n}\right)^{-3 / 2} \exp \left(-b_{n}^{+}\left(\mathbf{p}_{1}+\mathbf{p}_{2}\right)^{2}-b_{n}^{-}\left(\mathbf{p}_{1}-\mathbf{p}_{2}\right)^{2}\right), \\
g_{n} & =\left(8 \Delta^{2} A_{n} b_{n}^{+}\right)^{-3 / 2},
\end{aligned}
$$

where $A_{n}, b_{n}^{+}$and $b_{n}^{-}$are given by the recurrence relations:

$$
\begin{aligned}
A_{n} & =2 \Delta^{2} A_{n-1}\left(b_{n-1}^{+}+b_{n-1}^{-}+b_{1}^{+}+b_{1}^{-}\right), \\
1 / b_{n}^{+} & =1 /\left(b_{n-1}^{+}+b_{1}^{+}\right)+1 /\left(b_{n-1}^{-}+b_{1}^{-}\right), \\
b_{n}^{-} & =b_{1}^{+} b_{1}^{-} / b_{n}^{+},
\end{aligned}
$$

with $A_{1}=1, b_{1}^{+}=1 /\left(8 \Delta^{2}\right)$ and $b_{1}^{-}=r_{0}^{2} / 2$. The recurrence relations of this type allow for the analytical solution [17]. In our case it reads as:

$$
b_{n}^{+}=b_{1}^{+} \epsilon^{-1}\left(1-\rho^{n}\right) /\left(1+\rho^{n}\right), \quad A_{n}=\beta^{2 n / 3} \epsilon\left(1-\rho^{2 n}\right), \quad g_{n}=\beta^{-n}\left(1-\rho^{n}\right)^{-3},
$$

where $\epsilon^{-1}=2 r_{0} \Delta, \rho=(1-\epsilon) /(1+\epsilon)$ and the parameter

$$
\beta=\left(r_{0} \Delta+1 / 2\right)^{3}
$$

can be considered as a characteristic phase space volume.

For example, for $n=2$ and 3, we have: $A_{2}=\left(1+\epsilon^{-2}\right) / 2, A_{3}=\left(1+3 \epsilon^{-2}\right)^{2} / 16, b_{2}^{+}=2 b_{1}^{+} /\left(1+\epsilon^{2}\right), b_{3}^{+}=$ $3 b_{1}^{+}\left(1+\epsilon^{2} / 3\right) /\left(1+3 \epsilon^{2}\right), g_{2}=\epsilon^{3}, g_{3}=\left[4 \epsilon^{2} /\left(3+\epsilon^{2}\right)\right]^{3}$. Recall that the cumulants related to $g_{2}$ and $g_{3}$ are (see Eq. (25) $K_{2}=g_{2}$ and $K_{3}=2 g_{3}$.

It follows from the recurrence relations (50) or their analytical solutions (51) that the slope parameters $b_{n}^{+}$and $b_{n}^{-}$ approach each other with increasing $n$. In the large- $n\left(n>r_{0} \Delta\right)$ limit we then have 15$]$ :

$$
b_{n}^{+} \rightarrow b_{n}^{-} \rightarrow r_{0} /(4 \Delta), \quad A_{n} \rightarrow \beta^{2 n / 3} /\left(2 r_{0} \Delta\right), \quad g_{n} \rightarrow \beta^{-n}
$$

and

$$
G_{n}\left(\mathbf{p}_{1}, \mathbf{p}_{2}\right) \rightarrow \beta^{-n}\left(\frac{r_{0}}{\pi \Delta}\right)^{3 / 2} \exp \left(-\frac{r_{0}}{4 \Delta}\left(4 \mathbf{p}^{2}+\mathbf{q}^{2}\right)\right) .
$$

In very large- $n(n>e \beta)$ limit, using the large- $n$ behavior of the parameters $g_{n}$, we can get from the recurrence relation (26) the following behavior of the BE weight 15]:

$$
\omega_{n} \rightarrow c(\beta) n ! / \beta^{n},
$$

where $c(\beta)$ is a function factorially increasing with $\beta, c(1)=1$.

It is worth noting that the large- $n$ limits become equalities at $\beta=1\left(r_{0} \Delta=1 / 2\right)$ when $g_{n}=A_{n}=1, K_{n}=(n-1)$ !, $\omega_{n}=n$ ! and $b_{n}^{-}=b_{n}^{+}=r_{0} /(4 \Delta)$. Recall that $\beta=1$ corresponds to the minimal possible phase space volume when all the particle emitters are situated at one and the same space-time point so that the size $(2 \Delta)^{-1}$ of the elementary source determines not only the width of the single-particle spectrum but also the characteristic distance between the production points (see however the footnote after Eq. (26) and also ref. [18 for a more detailed discussion). In such a case $\widetilde{r}_{0}=0$ and the correlation function equals 2 for any value of $\mathbf{q}$.

In the low- $n\left(n<r_{0} \Delta\right)$ limit, i.e. in the case of a large phase space volume, it follows from Eqs. (50) or (51) that the slope parameter $b_{n}^{+}$increases linearly with $n$ up to the corrections $O\left(\left(2 r_{0} \Delta\right)^{-2}\right)$ and that, at $n>2 r_{0} \Delta$, this increase saturates at $r_{0} /(4 \Delta)$. Similar behavior shows the parameter $A_{n} / \beta^{2 n / 3}$. Thus, at $n \ll 2 r_{0} \Delta$, we have:

\footnotetext{
${ }^{5} \mathrm{~A}$ good approximation is $c(\beta) \doteq \beta^{d(\beta)}, d(\beta)=a_{1}+a_{2} \beta^{a_{3}}, a_{1}=0.617, a_{2}=0.621$ and $a_{3}=0.788$.
} 


$$
b_{n}^{+}=b_{1}^{+} b_{1}^{-} / b_{n}^{-} \doteq n b_{1}^{+}, \quad A_{n} \doteq n\left(r_{0} \Delta\right)^{2(n-1)}, \quad g_{n} \doteq n^{-3}\left(r_{0} \Delta\right)^{-3(n-1)} .
$$

Comparing the low- $n$ approximations (56) for the parameters $b_{n}^{+}, A_{n}$ and $g_{n}$ with the large- $n$ ones in Eqs. (53), we can see that they tail each other at $n=2 r_{0} \Delta, r_{0} \Delta / 2$ and $r_{0} \Delta$ respectively. Correspondingly, the low- $n$ approximation for the $G_{n}$-function

$$
G_{n}\left(\mathbf{p}_{1}, \mathbf{p}_{2}\right) \doteq r_{0}^{3}\left(r_{0} \Delta\right)^{-3 n}(2 \pi n)^{-3 / 2} \exp \left(-\mathbf{p}^{2} n / 2 \Delta^{2}-\mathbf{q}^{2} r_{0}^{2} / 2 n\right)
$$

tails with the large- $n$ one in Eq. (54) at $n=n_{t}, 1 / 2<n_{t} /\left(r_{0} \Delta\right)<2$.

Consider now the correlation function $R_{n}$ defined in Eq. (35). To determine the normalization constant $c_{n}$, it is convenient to rewrite the single- and two-boson spectra at a fixed multiplicity $n$ as

$$
\begin{gathered}
N_{n}^{(1)}(\mathbf{p})=\sum_{j=0}^{n-1} \frac{\omega_{n-1-j} /(n-1-j) !}{\omega_{n} / n !} G_{j+1}(\mathbf{p}, \mathbf{p}) \equiv \sum_{j=0}^{n-1} \frac{w(n-1-j)}{w(n)} \widetilde{G}_{j+1}(\mathbf{p}, \mathbf{p}), \\
N_{n}^{(2)}\left(\mathbf{p}_{1}, \mathbf{p}_{2}\right)=\sum_{j=0}^{n-2} \frac{\omega_{n-2-j} /(n-2-j) !}{\omega_{n} / n !} \sum_{l=0}^{j}\left[G_{l+1}\left(\mathbf{p}_{1}, \mathbf{p}_{1}\right) G_{j-l+1}\left(\mathbf{p}_{2}, \mathbf{p}_{2}\right)+\right. \\
\left.G_{l+1}\left(\mathbf{p}_{1}, \mathbf{p}_{2}\right) G_{j-l+1}\left(\mathbf{p}_{2}, \mathbf{p}_{1}\right)\right] \\
\equiv \sum_{j=0}^{n-2} \frac{w(n-2-j)}{w(n)} \sum_{l=0}^{j}\left[\widetilde{G}_{l+1}\left(\mathbf{p}_{1}, \mathbf{p}_{1}\right) \widetilde{G}_{j-l+1}\left(\mathbf{p}_{2}, \mathbf{p}_{2}\right)+\widetilde{G}_{l+1}\left(\mathbf{p}_{1}, \mathbf{p}_{2}\right) \widetilde{G}_{j-l+1}\left(\mathbf{p}_{2}, \mathbf{p}_{1}\right)\right],
\end{gathered}
$$

where $w(n)$, defined in Eq. (29), coincides with the BE affected multiplicity distribution arising from the Poissonian one characterized by the initial mean multiplicity $\eta$ and $\widetilde{G}_{i}\left(\mathbf{p}_{1}, \mathbf{p}_{2}\right)=\eta^{i} G_{i}\left(\mathbf{p}_{1}, \mathbf{p}_{2}\right)$. Noting further that $b_{n}^{+}$approaches the limiting value $r_{0} /(4 \Delta)$ from below, while $b_{n}^{-}$does it from above, we can see from Eq. (49) that, at large $q$, all terms in Eqs. (58) for $N_{n}^{(1)}\left(\mathbf{p}_{1,2}\right)$ and $N_{n}^{(2)}\left(\mathbf{p}_{1}, \mathbf{p}_{2}\right)\left(\mathbf{p}_{1,2}=\mathbf{p} \pm \mathbf{q} / 2\right)$ can be neglected except for those containing the lowest slope $b_{1}^{+}$. For the normalization constant $c_{n}=\lim _{q \rightarrow \infty}\left[N_{n}^{(1)}\left(\mathbf{p}_{1}\right) N_{n}^{(1)}\left(\mathbf{p}_{2}\right) / N_{n}^{(2)}\left(\mathbf{p}_{1}, \mathbf{p}_{2}\right)\right]$ in Eq. (35) for the correlation function we thus get [15]:

$$
c_{n}=[w(n-1)]^{2} /[w(n) w(n-2)] \equiv n \omega_{n-1}^{2} /\left[(n-1) \omega_{n} \omega_{n-2}\right] .
$$

Note that $c_{2}=2 / \omega_{2} \in(1,2)$; with the increasing multiplicity $c_{n}$ decreases and, according to Eq. (55), $c_{n} \doteq 1$ for $n>e \beta$. For large phase space volumes (when $\omega_{n} \doteq 1$ at small $n$ ), the normalization $c_{n} \doteq n /(n-1)$ and the exclusive correlation function $R_{n}$, normalized to 1 at large $|\mathbf{q}|$, becomes close to the usual definition as a ratio of the two-particle spectrum to the product of the single-particle ones, both spectra normalized to 1 . Generally, the latter definition is however not reliable since it leads to the plateau height of $\omega_{n} \omega_{n-2} / \omega_{n-1}^{2}>1$ which, in case of a small phase space volume $\beta$ and a small $n$, can be substantially larger than 1 . For example, for $n=2$ this height is $\omega_{2}$ and can reach a value of 2 if $\beta \rightarrow 1$.

Regarding the (semi-)inclusive single- and two-boson spectra, they can be written in a form similar to Eqs. (58) only in the initially Poissonian case:

$$
\begin{aligned}
& N^{(1)}(\mathbf{p})=\sum_{n} \sum_{j=0}^{n-1} w(n-1-j) \widetilde{G}_{j+1}(\mathbf{p}, \mathbf{p}) / \sum_{n} w(n), \\
& N^{(2)}\left(\mathbf{p}_{1}, \mathbf{p}_{2}\right)=\sum_{n} \sum_{j=0}^{n-2} w(n-2-j) \sum_{l=0}^{j}\left[\widetilde{G}_{l+1}\left(\mathbf{p}_{1}, \mathbf{p}_{1}\right) \widetilde{G}_{j-l+1}\left(\mathbf{p}_{2}, \mathbf{p}_{2}\right)+\right. \\
&\left.\widetilde{G}_{l+1}\left(\mathbf{p}_{1}, \mathbf{p}_{2}\right) \widetilde{G}_{j-l+1}\left(\mathbf{p}_{2}, \mathbf{p}_{1}\right)\right] / \sum_{n} w(n) .
\end{aligned}
$$

The normalization constant in Eq. (36) for the (semi-)inclusive correlation function is then 15:

$$
c=\left[\sum_{n} w(n-1)\right]^{2} /\left[\sum_{n} w(n) \sum_{n} w(n-2)\right] .
$$

Clearly, in the completely inclusive case (when the sums include all $n$ from 0 to $\infty$ and $\sum_{n} w(n-j)=1$ ), we have $c=1$ and

$$
\langle n\rangle=\sum_{j=0}^{\infty} \widetilde{g}_{j+1} \equiv \widetilde{g},
$$




$$
\begin{aligned}
N^{(1)}(\mathbf{p}) & =\sum_{j=0}^{\infty} \widetilde{G}_{j+1}(\mathbf{p}, \mathbf{p}) \equiv \widetilde{G}(\mathbf{p}, \mathbf{p}) \\
N^{(2)}\left(\mathbf{p}_{1}, \mathbf{p}_{2}\right) & =\sum_{j=0}^{\infty} \sum_{l=0}^{j}\left[\widetilde{G}_{l+1}\left(\mathbf{p}_{1}, \mathbf{p}_{1}\right) \widetilde{G}_{j-l+1}\left(\mathbf{p}_{2}, \mathbf{p}_{2}\right)+\widetilde{G}_{l+1}\left(\mathbf{p}_{1}, \mathbf{p}_{2}\right) \widetilde{G}_{j-l+1}\left(\mathbf{p}_{2}, \mathbf{p}_{1}\right)\right] \\
& \equiv \widetilde{G}\left(\mathbf{p}_{1}, \mathbf{p}_{1}\right) \widetilde{G}\left(\mathbf{p}_{2}, \mathbf{p}_{2}\right)+\widetilde{G}\left(\mathbf{p}_{1}, \mathbf{p}_{2}\right) \widetilde{G}\left(\mathbf{p}_{2}, \mathbf{p}_{1}\right)
\end{aligned}
$$

where $\widetilde{g}_{n}=\eta^{n} g_{n}$. For the inclusive correlation function (36) we have [15]:

$$
R\left(\mathbf{p}_{1}, \mathbf{p}_{2}\right)=1+\frac{\widetilde{G}\left(\mathbf{p}_{1}, \mathbf{p}_{2}\right) \widetilde{G}\left(\mathbf{p}_{2}, \mathbf{p}_{1}\right)}{\widetilde{G}\left(\mathbf{p}_{1}, \mathbf{p}_{1}\right) \widetilde{G}\left(\mathbf{p}_{2}, \mathbf{p}_{2}\right)}
$$

Thus, in the considered case of the initially Poissonian multiplicity distribution, the intercept $R(\mathbf{p}, \mathbf{p}) \equiv R(0)=2$ in agreement with the result generally valid for thermalized systems [8]. Note that Eq. (63) coincides with similar expressions in refs. 16. 17] up to a normalization factor $\langle n\rangle^{2} /\langle n(n-1)\rangle$. With the increasing density, the latter decreases from 1 to $1 / 2$ and, for dense systems, forces the corresponding correlation function to 1 . Such a behavior was incorrectly interpreted [16, 17] as a coherent effect (see also a discussion in Section VI).

\section{RESULTS}

\section{A. Multiplicity distributions}

We will consider here the multiplicity distribution (29) resulting due to the BE effect on the initially Poissonian one with the mean multiplicity $\eta$. In accordance with the large- $n$ behavior of the BE weights in Eq. (55), it takes on the following limiting form at $n>e \beta[15$ :

$$
w(n) \rightarrow \text { const }^{\prime} \cdot \xi^{n}, \quad \xi=\eta / \beta .
$$

The large- $n$ behavior of the multiplicity distribution in Eq. (64) indicates that it approaches the BE one:

$$
w_{\mathrm{BE}}(n)=\nu^{n} /(1+\nu)^{n+1}, \quad \nu=\xi /(1-\xi),
$$

with the mean multiplicity $\nu$. . This is demonstrated in Figs. 1 and 2 . Thus, at $r_{0}=2.1 \mathrm{fm}$ and $\Delta=0.25 \mathrm{GeV} / \mathrm{c}$, the BE effect transforms the initial Poissonian multiplicity distribution with $\eta=30$ (dotted curve in Fig. 1a) to the one with much higher mean and dispersion values (solid curve in Fig. 1a). The exponential tail expected for the BE distribution is clearly seen in Fig. $1 \mathrm{~b}$ where the results are presented in logarithmic scale for $\eta=10, \Delta=0.25 \mathrm{GeV} / \mathrm{c}$ and $r_{0}=1.5 \mathrm{fm}$. One may see that Eq. (64) (dashed line) becomes an excellent approximation for $n>30$, which is close to the condition $n>e \beta=37.6$ for the present choice of parameters. The slope parameter $b$ in the exponential fit $w(n)=$ const $\cdot \exp (-b n)$ of this tail at large $n$ should be, according to Eq. (64), only a function of the variable $\xi$ : $b=-\ln (\xi)$. Such a scaling is demonstrated in Fig. 2a for various values of $\eta, \Delta$ and $r_{0}$. Note that $\xi=0.95$ and 0.72 for Figs. 1a and 1b, corresponding to $b=0.02$ and 0.27 , respectively 7

It should be noted that the experimental data point to a moderate value of the density parameter $\xi$. Thus, taking 0.2 as an estimate of the inclusive phase space density at $\mathbf{p}=0$ from AGS and SPS experiments and using Eq. (47), we get (see the last Section) $\xi \approx 0.4-0.5$.

\footnotetext{
${ }^{6}$ This is in accordance with the appearance of the Bose-condensate in a dense ideal Bose gas [24. The fluctuations of the number of particles in the condensate are very large - they are described by the well-known Einstein formula for identical bosons in the same quantum state. The corresponding BE multiplicity distribution in Eq. (65) turns to the Reley one for very large mean multiplicities. This type of BE condensate should not be mixed up with the multiboson coherent (laser) state in which the BE correlations are absent and the multiplicity distribution corresponds to the Poisson law.

${ }^{7}$ At the explosion point $\xi=1$ the tail of the multiplicity distribution becomes a constant $(b=0)$ so that the mean multiplicity $\langle n\rangle$ would go to infinity provided that there are no energy-momentum constraints. Note that the corresponding critical initial mean multiplicity $\eta_{c r}=\beta \equiv\left(r_{0} \Delta+1 / 2\right)^{3}$ is close but different from that given in Eq. (9) of ref. [5]. For the origin of this difference see discussion in Section $\mathrm{VI}$.
} 
Using Eq. (62) for $\langle n\rangle$ and tailing the large- and small- $n$ approximations of the integrals $g_{n}$ at $n_{t}=r_{0} \Delta$ (see Eqs. (53) and (56) ), we can approximate the mean multiplicity as

$$
\begin{aligned}
\langle n\rangle & \doteq\left[1+\tilde{\xi} / 2^{3}+\cdots+\tilde{\xi}^{n_{t}-1} / n_{t}^{3}\right] \eta+\xi^{n_{t}} \nu \\
& \equiv\langle n\rangle_{\mathrm{g}}+\langle n\rangle_{\mathrm{c}}
\end{aligned}
$$

where $\tilde{\xi}=\eta /\left(r_{0} \Delta\right)^{3}>\xi$; the density parameters $\tilde{\xi}$ and $\xi$ coincide at $r_{0} \Delta \gg 1$. At large phase space volumes, $\left(r_{0} \Delta\right)^{3} \gg 1$, the two terms in Eq. (66) can be considered as contributions of the BE gas and BE condensate respectively. It can be seen that the condensate dominates on condition $\langle n\rangle>\beta$.

Note that in the rare gas limit $\tilde{\xi} \ll 1$, we have $\langle n\rangle \doteq\left[1+\tilde{\xi} / 2^{3}\right] \eta \equiv \eta+K_{2} \eta^{2}$, i.e. the increase of the mean multiplicity is dominated by the contribution of the second order cumulant $K_{2}=\left(2 r_{0} \Delta\right)^{-3}$. The corresponding multiplicity distribution then becomes somewhat wider than the Poissonian one (see Eqs. (27), (29)): $w(n)=$ const $\cdot\left(1+C_{2}^{n} K_{2}\right) \eta^{n} / n !$.

In Fig. 2c we demonstrate the approach of the mean multiplicity $\langle n\rangle$ to the limiting scaling value $\nu=\xi /(1-\xi)$, though only for $\xi$ very close to the explosion point $\xi=1(\xi>0.99)$. Instead, in the region of $\xi<0.9$ indicated by present experiments, we can see, in agreement with Eq. (66) an approximate $\xi$-scaling of the ratio $\langle n\rangle / \eta($ Fig. $2 \mathrm{~b})$.

Since, in the realistic event generators, the multiboson effects can be accounted for only in the lowest order cumulant approximation 18, it is instructive to study the saturation of these effects with the increasing number $N_{c u m}$ of the contributing cumulants. In Fig. 3 we show $N_{c u m}$-dependence of the ratio $\langle n\rangle / \eta$ of the BE affected mean multiplicity to the initial one for different values of the density parameter $\xi$. For example, at $\xi=0.8$ this ratio saturates at $N_{\text {cum }} \sim 10(\sim 40 \%$ increase of $\langle n\rangle)$. At $N_{\text {cum }}=4$, representing a practical limit due to the numerical problems [18], the effect is underestimated by $\sim 25 \%(\langle n\rangle / \eta \approx 1.3$ instead of 1.4$)$. The situation is more optimistic for lower densities. Thus at $\xi=0.5$ the effect $(\sim 15 \%$ increase of $\langle n\rangle)$ practically saturates at $N_{\text {cum }}=4$.

Up to now, we have considered the symmetrization effect on the production of only one type of identical bosons. For a system of charged and neutral pions, the total symmetrization weight in the model coincides with a product of the separate BE weights $\omega_{n_{+}}, \omega_{n_{-}}$and $\omega_{n_{0}}$ provided the pions are emitted in unpolarized and uncorrelated isospin states, the effect of their FSI is negligible and there are practically no restrictions due to energy-momentum and isospin constraints. The latter assumption may be reasonable at high energies when the subsystem of interfering pions represents a small part of the produced multiparticle system. The initial distribution of pion species is then a trinomial one, strongly peaked at $n_{+}=n_{-}=n_{0}=n / 3: w\left(n_{+}, n_{-}, n_{0}\right)=n ! /\left(3^{n} n_{+} ! n_{-} ! n_{0} !\right)$. After the symmetrization in the large $-n$ condensate limit $\left(\omega_{n_{i}} \doteq n_{i} ! / \beta^{n_{i}}\right)$, it becomes independent of $n_{i}$ and yields a substantial probability $\left(2\left(n-n_{0}+1\right) /[(n+1)(n+2)]\right)$ of any value of $n_{0}$ at a fixed total pion multiplicity $n$. Particularly, the production of so called Centauro (anti-Centauro) events containing mainly charged (neutral) pions then becomes possible. The probability of the extreme charge configurations can be enhanced even stronger in case of isospin constraints, for example, if pions were produced in isosinglet pairs [5, 6, 25. The latter mechanism can be of particular importance in the near-threshold multipion production, $\$$ due to the limited total isospin and charge. Unfortunately, the $n$ ! enhancement of the near-threshold condensate production will be more than compensated by the phase space suppression factor of $\left(\bar{p}_{n} / \Delta\right)^{3 n} \sim(n !)^{-3 / 2}$, where $\bar{p}_{n}=\left[2 m\left(\sqrt{s}-\sum_{i} m_{i}\right) / n\right]^{1 / 2}$ is the mean pion momentum near threshold.

\section{B. Single-particle spectra}

The influence of the BE effect on the single-boson spectrum for a given boson multiplicity $n$, can be seen from Eqs. (25)-(30), (32) and (49). At sufficiently large momenta, when the local density $\left\langle f_{n}\right\rangle_{\mathbf{p}}$ remains small even at large $n$, this spectrum is dominated by the contribution $\beta_{n} P(\mathbf{p}), \beta_{n}=n \omega_{n-1} / \omega_{n}$, of the initial spectrum. In such a rare gas limit, $\xi_{n, \mathbf{p}} \equiv \xi_{n} \exp \left(-\mathbf{p}^{2} / 2 \Delta^{2}\right) \ll 1$, we can write (see Eqs. (25), (32) and (27)):

$$
\begin{aligned}
N_{n}(\mathbf{p}) & \doteq n \frac{\omega_{n-1}}{\omega_{n}} G_{1}(\mathbf{p}, \mathbf{p})+n(n-1) \frac{\omega_{n-2}}{\omega_{n}} G_{2}(\mathbf{p}, \mathbf{p}) \\
& \doteq n\left[1-(n-1) K_{2}\right] G_{1}(\mathbf{p}, \mathbf{p})+n(n-1) G_{2}(\mathbf{p}, \mathbf{p}) \\
& \doteq n P(\mathbf{p})+n(n-1) K_{2}\left[2^{3 / 2} P\left(2^{1 / 2} \mathbf{p}\right)-P(\mathbf{p})\right]
\end{aligned}
$$

\footnotetext{
${ }^{8}$ A proposal of an experimental study of the near-threshold multipion system at Serpukhov accelerator was recently discussed by V.A. Nikitin. Similar idea was also communicated to us by L.L. Nemenov.
} 
Otherwise, at large local densities, $N_{n}(\mathbf{p})$ is determined by the asymptotic large-density spectrum 15:

$$
N_{n}(\mathbf{p}) \rightarrow n\left(\frac{r_{0}}{\pi \Delta}\right)^{3 / 2} \exp \left(-\frac{r_{0}}{\Delta} \mathbf{p}^{2}\right) \equiv n P_{\mathrm{c}}(\mathbf{p}),
$$

associated with the BE condensate and corresponding to the asymptotic (large- $n$ ) value $r_{0} /(4 \Delta)$ of the slope parameters $b_{n}^{ \pm}$. Note that $P_{\mathrm{c}}(\mathbf{p})$ is normalized to unity and that, at $\beta=1$, it coincides with the initial distribution $P(\mathbf{p})$.

It is clear from Eqs. (54), (55) and (58) that for small momenta, $p<\Delta\left(r_{0} \Delta-1 / 2\right)^{-1 / 2}$, the condensate regime in Eq. (68) settles on condition $\xi_{n}>e$. For larger momenta, we must take into account that the condensate contribution vanishes much faster than that of $\mathrm{BE}$ gas, thus leading to much stronger condition of the condensate dominance:

$$
\xi_{n}>\left(2 r_{0} \Delta\right)^{-3 / 2} \exp \left[\left(2 r_{0} \Delta-1\right) p^{2} / 2 \Delta^{2}\right] .
$$

Similar to the fixed multiplicity case, the inclusive single-boson spectrum at small local densities tends to $\eta P(\mathbf{p}) \equiv$ $\widetilde{G}_{1}(\mathbf{p}, \mathbf{p})$ and, at large ones, it approaches the asymptotic high-density spectrum (see Eq. (68)):

$$
N(\mathbf{p}) \rightarrow\langle n\rangle P_{\mathrm{c}}(\mathbf{p})
$$

The transfer of the initial spectrum to the high-density one is demonstrated for the inclusive distribution in Fig. 4a and, more clearly, for $\xi$ closer to the explosion point $\xi=1$, in Fig. $4 \mathrm{~b}$.

Tailing the large- and small- $n$ behavior of the $G_{n}$-functions at $n_{t} \sim r_{0} \Delta$ (see Eqs. (54) and (57)), we can approximate the inclusive single-boson spectrum in Eq. (62) as

$$
\begin{aligned}
N(\mathbf{p}) & \doteq\left[1+\tilde{\xi}_{\mathbf{p}} / 2^{3 / 2}+\cdots+\tilde{\xi}_{\mathbf{p}}^{n_{t}-1} / n_{t}^{3 / 2}\right] \eta P(\mathbf{p})+\xi^{n_{t}} \nu P_{\mathrm{c}}(\mathbf{p}) \\
& \equiv N_{\mathrm{g}}(\mathbf{p})+N_{\mathrm{c}}(\mathbf{p}),
\end{aligned}
$$

where $\tilde{\xi}_{\mathbf{p}}=(2 \pi)^{3 / 2} \eta P(\mathbf{p}) / r_{0}{ }^{3} \equiv \tilde{\xi} \exp \left(-\mathbf{p}^{2} / 2 \Delta^{2}\right)$. Clearly, for large phase space volumes, $\left(r_{0} \Delta\right)^{3} \gg 1$, the two terms in Eq. (71) can be interpreted as contributions of the BE gas and BE condensate respectively. Like in the fixed multiplicity case, the condensate dominates on condition (69) with the substitution $n \rightarrow\langle n\rangle\left(\xi_{n} \rightarrow\langle n\rangle / \beta\right)$.

In Fig. 4 we compare the inclusive single-boson spectra with the approximate formula (71). A good agreement is obtained despite the calculations were done for not very large phase space volumes. Some underestimation of $N(\mathbf{p})$ at intermediate local densities $\widetilde{\xi}_{\mathbf{p}}$ (Fig. 4a) and the corresponding underestimation of $\langle n\rangle$ at moderate densities $\xi$ (Fig. 2b) become weaker for larger systems (larger multiplicities in Fig. 2b) due to increasing number $n_{t} \sim r_{0} \Delta$ of the terms in Eqs. (66), (71) and thus - decreasing relative contribution of the tailing region. We may conclude that the accuracy of Eqs. (66) and (71) is reasonable for the systems produced in heavy ion collisions at SPS and that it will be even better for larger systems at RHIC and LHC. Experimentally the effect of BE "condensate" was searched for at SPS CERN as a low- $p_{t}$ enhancement, however, with rather uncertain results (see, e.g., 26]).

It follows from Eq. (71) that for sufficiently large $\left(n_{t}=r_{0} \Delta \gg 1\right)$ and not very dense $(\xi \ll 1)$ systems, similarly to the $\xi$-scaling of $\langle n\rangle / \eta$ (see Fig. $2 \mathrm{~b}$ ), the ratio $N(\mathbf{p}) /[\eta P(\mathbf{p})]$ scales with the local density parameter $\tilde{\xi}_{\mathbf{p}}$. It appears that analogical scaling takes place also at fixed multiplicity $n . P$ In Fig. 5 we show the ratio of the single-particle spectrum at fixed $n$ to the dominant large-p contribution $\beta_{n} P(\mathbf{p})$ of the initial spectrum calculated at $\mathbf{p}=0$ as a function of $\xi_{n}$ for various multiplicities $n$. An approximate $\xi_{n}$-scaling is seen up to $\xi_{n}$ of the order of unity. At larger $\xi_{n}$ this ratio approaches the condensate limit $\left(2 r_{0} \Delta\right)^{3 / 2} \xi_{n}$ which no more scales with $\xi_{n}$ (see the corresponding curves in Fig. 5b). What scales at large $\xi_{n}$ is not the ratio of the two contributions but the ratio of their integrals, $n / \beta_{n}$, the limiting value of which is just equal to $\xi_{n}=n / \beta$ since, according to Eq. (55), $\beta_{n}=n \omega_{n-1} / \omega_{n} \rightarrow \beta$ for $n>\mathrm{e} \beta$.

Note that, in the absence of simple analytical approximations for the fixed- $n$ spectra, the approximate $\xi_{n^{-}}$or $\xi_{n, \mathbf{p}^{-}}$ scaling can be used to overcome technical problems with factorially large numbers at high multiplicities. For example, $N_{n}(0)$ at a given density $\xi_{n}<1$ can be obtained by calculating $N_{n^{\prime}}(0)$ at a smaller multiplicity $n^{\prime}<n$ keeping the same density $\xi_{n^{\prime}}=\xi_{n}$ and then rescaling to $N_{n}(0) \doteq\left(\beta_{n} / \beta_{n^{\prime}}\right) N_{n^{\prime}}(0)$, where $\beta_{n} / \beta_{n^{\prime}} \doteq 1$ for $n^{\prime}>e \beta$.

\footnotetext{
${ }^{9}$ These results agree with those obtained in refs. [5.6] (see also [8, 18] and references therein) except for an incorrect conclusion [月. that the width of the narrow peak due to the $\mathrm{BE}$ " condensate" is of $1 / r_{0}$.

${ }^{10}$ In this case, due to the explicit dependence of the particle spectra on the complicated BE weights $\omega_{n}$, there is no analytical approximation similar to Eq. (71).
} 


\section{Correlation functions}

It follows from Eqs. (35) and (49)-(59) that, for a given multiplicity $n$, the correlation function intercept $R_{n}(\mathbf{p}, \mathbf{p}) \equiv$ $R_{n}(0)$ decreases and the correlation function width increases with the increasing $n$ or decreasing momentum $p$, both corresponding to the increasing local density parameter $\xi_{n, \mathbf{p}}$.

In fact, for large local densities (see Eq. (69)), the condensate behavior is achieved (see the curves in Fig. 5b) and the correlation function tends to unity not only at large but also at small $\mathbf{q}^{2}$. Indeed, in this limit the normalization constant $c_{n} \rightarrow 1$ (see Eqs. (38) and (55)) and the nominator and denominator of the correlation function (35) consist of about the same number $\sim n^{2}$ of the condensate terms $\left(r_{0} / \pi \Delta\right)^{3} \exp \left[-\left(4 \mathbf{p}^{2}+\mathbf{q}^{2}\right) r_{0} / 2 \Delta\right]$ (see Eqs. (54), (55) and (58)) ${ }^{1]}$ The well known [3 5 ] lowering and widening of the correlation function with the increasing multiplicity is demonstrated, in the considered model, in Fig. 6.

Note however that in the rare gas limit, $\xi_{n, \mathbf{p}} \ll 1$, the change of the form of the correlation function with the increasing density is rather weak. Thus, writing in this limit

$$
\begin{array}{r}
N_{n}^{(2)}\left(\mathbf{p}_{1}, \mathbf{p}_{2}\right) \doteq n(n-1) \frac{\omega_{n-2}}{\omega_{n}}\left[G_{1}\left(\mathbf{p}_{1}, \mathbf{p}_{1}\right) G_{1}\left(\mathbf{p}_{2}, \mathbf{p}_{2}\right)+G_{1}\left(\mathbf{p}_{1}, \mathbf{p}_{2}\right) G_{1}\left(\mathbf{p}_{2}, \mathbf{p}_{1}\right)\right] \\
+n(n-1)(n-2) \frac{\omega_{n-3}}{\omega_{n}}\left[G_{1}\left(\mathbf{p}_{1}, \mathbf{p}_{1}\right) G_{2}\left(\mathbf{p}_{2}, \mathbf{p}_{2}\right)+G_{1}\left(\mathbf{p}_{1}, \mathbf{p}_{2}\right) G_{2}\left(\mathbf{p}_{2}, \mathbf{p}_{1}\right)\right. \\
\left.+G_{2}\left(\mathbf{p}_{1}, \mathbf{p}_{1}\right) G_{1}\left(\mathbf{p}_{2}, \mathbf{p}_{2}\right)+G_{2}\left(\mathbf{p}_{1}, \mathbf{p}_{2}\right) G_{1}\left(\mathbf{p}_{2}, \mathbf{p}_{1}\right)\right] \\
\doteq n(n-1)\left[1-(2 n-3) K_{2}\right] P\left(\mathbf{p}_{1}\right) P\left(\mathbf{p}_{2}\right)\left[1+\exp \left(-\tilde{r}_{0} \mathbf{q}^{2}\right)\right] \\
+n(n-1)(n-2) 2^{3 / 2} K_{2}\left\{P\left(\mathbf{p}_{1}\right) P\left(2^{1 / 2} \mathbf{p}_{2}\right)\left[1+\exp \left(-\frac{3}{4} \tilde{r}_{0}{ }^{2} \mathbf{q}^{2}+\frac{\mathbf{p q}}{2 \Delta^{2}}\right)\right]\right. \\
\left.+P\left(\mathbf{p}_{2}\right) P\left(2^{1 / 2} \mathbf{p}_{1}\right)\left[1+\exp \left(-\frac{3}{4} \tilde{r}_{0}{ }^{2} \mathbf{q}^{2}-\frac{\mathbf{p q}}{2 \Delta^{2}}\right)\right]\right\},
\end{array}
$$

$\tilde{r}_{0}^{2}=r_{0}^{2}\left[1-\left(2 r_{0} \Delta\right)^{-2}\right]$, we get for the correlation function intercept an $n$-independent value close to $2: R_{n}(0) \doteq$ $2\left(1-\epsilon_{1}\right)$. Here we have introduced the density parameter

$$
\epsilon_{j}=j \cdot 2^{5 / 2} K_{2} \exp \left(-\mathbf{p}^{2} / 2 \Delta^{2}\right) \doteq 2^{-1 / 2} \xi_{j, \mathbf{p}} .
$$

It should be noted that the correlation function $R_{n}(\mathbf{q})$ becomes less than unity at intermediate q-values and approaches the limiting value of 1 from below. This behavior and also the related suppression of the intercept value is caused by the $\mathrm{BE}$ correlation effect on the single-particle spectra entering the denominator of the correlation function. Sometimes this distortion is corrected for by a special iterative procedure. Its result can be described by a simple low- $\mathbf{q}^{2}$ correction factor:12

$$
R_{n}^{c o r}\left(\mathbf{p}_{1}, \mathbf{p}_{2}\right) \doteq\left(A_{n}-B_{n} \mathbf{q}^{2}\right) R_{n}\left(\mathbf{p}_{1}, \mathbf{p}_{2}\right) .
$$

In the low density limit of our model, we have

$$
A_{n}=1+\epsilon_{n-1}, \quad B_{n}=\epsilon_{n-1}\left[1-(\mathbf{p} / \Delta)^{2} \cos ^{2} \psi\right] / 8 \Delta^{2},
$$

where $\psi$ is the angle between the vectors $\mathbf{p}$ and $\mathbf{q}$. At small $\mathbf{q}^{2}$, the corrected correlation function (properly normalized to unity at large $\mathbf{q}^{2}$ ) is then

$$
R_{n}^{c o r}\left(\mathbf{p}_{1}, \mathbf{p}_{2}\right) \doteq 1+\exp \left(-\tilde{r}_{0}^{2} \mathbf{q}^{2}\right)+\epsilon_{n-2}\left[1+\exp \left(-\frac{3}{4} \tilde{r}_{0}^{2} \mathbf{q}^{2}\right)\right]
$$

\footnotetext{
${ }^{11}$ Of course, the absence of the correlation in the condensate limit at fixed multiplicity has nothing to do with the coherence effect which is absent in the considered model. See the footnote after Eq. (26) and the discussion of the inclusive correlation function which appears to be different from 1 at whatever high densities.

${ }^{12}$ The iterative correction procedure is usually used for small-acceptance detectors triggered by the requirement of at least two identical pions in the detector. The mixed reference sample then differs from the product of the single-particle spectra, being much more influenced by the residual BE correlations. The residual correlations can substantially affect also single-particle spectra in the case of a small effective emission volume, e.g., in $e^{+} e^{-}$-collisions. There are also other reasons for the low- $q^{2}$ correction factor, like energy-momentum constraints or presence of dynamical correlations (e.g., in jets) which are destroyed in the mixed reference sample. For this reason the correction factor similar to that in Eq. (74) is often introduced as a pure phenomenological one with $A_{n}=1$ and $B_{n}$ treated as a free parameter.
} 
and can be represented in the usual single-Gaussian form: ${ }^{33}$

$$
\begin{gathered}
R_{n}^{c o r}\left(\mathbf{p}_{1}, \mathbf{p}_{2}\right) \doteq 1+\lambda_{n} \exp \left(-r_{n}{ }^{2} \mathbf{q}^{2}\right), \\
\lambda_{n}=1+2 \epsilon_{n-2}, \quad r_{n}{ }^{2}=\tilde{r}_{0}^{2}\left(1-\frac{5}{4} \epsilon_{n-2}\right) .
\end{gathered}
$$

We see that with the increasing $n$ the effective interferometry parameters $\lambda_{n}$ and $r_{n}$ respectively increase and decrease slightly, starting from the zero density values of 1 and $\tilde{r}_{0}$.

In the low-density limit, simple Eqs. (76), (77) or directly Eqs. (67) and (72) allow one to determine the radius parameter $r_{0}$ by fitting the correlation functions $R_{n}^{\text {cor }}$ or $R_{n}$. At higher densities, however, there is no simple analytical expression for the correlation function $R_{n}$ and the eventual fit would require the use of rather complicated Eqs. (58). Another possibility is still a simple single-Gaussian fit at sufficiently small q, giving the effective interferometry parameters $\lambda_{n}^{\text {eff }}<1$ and $r_{n}^{\text {eff }}<r_{0}$, both vanishing with the increasing local density. The low-density radius $r_{0}$ and the density can then be determined comparing $\lambda_{n}^{\text {eff }}$ and $r_{n}^{\text {eff }}$ with the model predictions as functions of $r_{0}$ and $\Delta$.

In Fig. 7 we show the intercept as a function of the multiplicity $n$ and the local density parameter $\xi_{n, \mathbf{p}}$ for several values of the mean momentum: $p=0,0.1,0.2$ and $0.4 \mathrm{GeV} / \mathrm{c}$. As expected, the intercept is practically constant at low local densities (small $n$ or high $p$ ). As condensate develops, the intercept sharply falls down. The sharpness of this drop is however less pronounced at higher momenta even if plotted as a function of the local density parameter $\xi_{n, \mathbf{p}}$. Clearly, this lack of density scaling is related to a strong decrease of the condensate contribution with the increasing momentum. In fact, for the momenta $p>\Delta\left(r_{0} \Delta-1 / 2\right)^{-1 / 2}$ the low-density parameter $\xi_{n, \mathbf{p}}$ strongly overestimates the local density in the region of the condensate dominance (see Eq. (69)). To demonstrate the possibility of the observation of the condensate effect for a speckle of a large number of soft pions not following the ordinary proportionality rule between the freeze-out phase space volume and pion multiplicity, in Fig. 7 we indicate by the arrows the intercept values corresponding to $\xi_{n}=3 \xi=1.5(n \approx 3\langle n\rangle)$.

In the inclusive case corresponding to the initial Poissonian multiplicity distribution, the correlation function intercept is equal to 2 for any local densities (see Eq. (63)). At very large local densities the two-boson spectrum approaches twice the product of the single-boson ones (see Eqs. (54) and (62)) so that the inclusive correlation function tends to the limiting value of 2 even at rather large relative momenta. The corresponding increase of the width of the correlation function with the increasing density parameter $\xi$ is demonstrated in Fig. 8.

Note that at high local densities both the nominator and denominator of the correlation function at small $\mathbf{q}^{2}$ are dominated, like in the case of a fixed multiplicity, by the universal condensate terms, their numbers being about $\langle n(n-1)\rangle$ and $\langle n\rangle^{2}$ respectively. The difference between the inclusive and fixed multiplicity correlation functions, $R \rightarrow 2$ and $R_{n} \rightarrow 1$, is due to the fact that at high densities the initially Poissonian multiplicity distribution approaches a much wider BE one, for which $\langle n(n-1)\rangle=2\langle n\rangle^{2}$.

Tailing the approximate equations (54) and (57) for the $G_{n}$-functions at $n_{t} \sim r_{0} \Delta$, we can analytically follow the behavior of the function $\widetilde{G}\left(\mathbf{p}_{1}, \mathbf{p}_{2}\right)$ (determining, according to Eq. (62), the inclusive two-boson spectrum $\left.N\left(\mathbf{p}_{1}, \mathbf{p}_{2}\right)\right)$ similar to Eq. (71), modifying it by the substitutions: $\widetilde{\xi}_{\mathbf{p}}^{n-1} \rightarrow \widetilde{\xi}_{\mathbf{p}}^{n-1} \exp \left(-\mathbf{q}^{2} r_{0}^{2} / 2 n\right)$ and $P_{c}(\mathbf{p}) \rightarrow P_{c}(\mathbf{p}) \exp \left(-\mathbf{q}^{2} r_{0} / 4 \Delta\right)$. Particularly, for large systems $\left(\left(2 r_{0} \Delta\right)^{2} \gg 1\right)$, we have at $q \rightarrow 0$ :

$$
\widetilde{G}\left(\mathbf{p}_{1}, \mathbf{p}_{2}\right) \doteq N_{\mathrm{g}}(\mathbf{p}) \exp \left(-\mathbf{q}^{2} r_{\mathrm{g}}{ }^{2} / 2\right)+N_{\mathrm{c}}(\mathbf{p}) \exp \left(-\mathbf{q}^{2} r_{\mathrm{c}}{ }^{2} / 2\right),
$$

where $r_{\mathrm{c}}^{2}=r_{0} /(2 \Delta)$ and

$$
r_{\mathrm{g}}{ }^{2} \doteq r_{0}{ }^{2} \sum_{n=1}^{n_{t}} \tilde{\xi}_{\mathbf{p}}{ }^{n} n^{-5 / 2} / \sum_{n=1}^{n_{t}} \tilde{\xi}_{\mathbf{p}}{ }^{n} n^{-3 / 2}
$$

Note that at low local densities $\left(\tilde{\xi}_{\mathrm{p}} \ll 1\right)$ the effective radius $r_{\mathrm{g}}$ coincides with $\tilde{r}_{0} \doteq r_{0}$. With the increasing local phase space density it slightly decreases. The maximal reduction factor of $1 / \sqrt{2}$ is achieved for large $\left(r_{0} \Delta \gg 1\right)$ and dense $(\xi \rightarrow 1, \mathbf{p} \rightarrow 0)$ systems. Considering the limit $q \rightarrow 0$ and $r_{0} \Delta \gg 1$, we can neglect the $q$-dependence of the condensate term $\left(r_{\mathrm{c}} \ll r_{\mathrm{g}}\right)$ and of the product of the single-particle spectra in the denominator of the correlation function and, using Eqs. (63) and (78), write

\footnotetext{
${ }^{13}$ Note that in [17] a similar parameterization was used for the uncorrected correlation function. This led to different estimates of the interferometry parameters $\lambda_{n}$ and $r_{n}$ in the considered model.
} 


$$
R\left(\mathbf{p}_{1}, \mathbf{p}_{2}\right) \doteq 1+\exp \left(-\frac{N_{\mathrm{g}}(\mathbf{p})}{N_{\mathrm{g}}(\mathbf{p})+N_{\mathrm{c}}(\mathbf{p})} r_{\mathrm{g}}{ }^{2} \mathbf{q}^{2}\right)
$$

It follows from Eq. (80) that the condensate contribution leads to an additional reduction of the interferometry radius squared (defined as a low- $\mathbf{q}^{2}$ slope of the correlation function) as compared with the case of a pure BE gas. In case of a dominant $\mathrm{BE}$ condensate the interferometry radius tends to zero whatever large is the geometric size of the system.

Note however that, due to the non-Gaussian character of the correlation functions at large phase space densities, their real width is determined by the large- $\mathbf{q}^{2}$ behavior which reads as:

$$
R\left(\mathbf{p}_{1}, \mathbf{p}_{2}\right) \doteq 1+\left(\frac{N_{\mathrm{c}}(\mathbf{p})}{N_{\mathrm{g}}(\mathbf{p})}\right)^{2} \exp \left(-r_{\mathrm{c}}^{2} \mathbf{q}^{2}\right)
$$

Requiring $R\left(\mathbf{q}_{\mathrm{eff}}\right)=1+1 / \mathrm{e}$, we get for the corresponding effective radius squared:

$$
r_{\mathrm{eff}}^{2} \equiv \mathbf{q}_{\mathrm{eff}}^{-2}={r_{\mathrm{c}}}^{2} /\left\{1+2 \ln \left[N_{\mathrm{c}}(\mathbf{p}) / N_{\mathrm{g}}(\mathbf{p})\right]\right\} .
$$

Thus, compared with the vanishing of the low- $\mathbf{q}^{2}$ slope of the correlation function (as $(1-\xi)$ ), the effective radius squared vanishes at large phase space densities much slower (as $1 /|\ln (1-\xi)|)$ (see Fig. \&).

It should be noted that Eqs. (62) and (63) assume that the initial Poissonian multiplicity distribution extends to any arbitrarily large number of bosons. In reality, however, this number is limited due to the finite available energy (for a study of the energy constraint effect on pion multiplicity see second paper in ref. [16]). It is therefore interesting to see how fast the semi-inclusive spectra approach the inclusive limit with the increasing number $n_{\max }$ of the included pions. In Fig. 9 we demonstrate the $n_{\max }$-dependence of the semi-inclusive correlation functions for a fixed value of the density parameter $\xi=0.95$ and, in Fig. 10 - the $n_{\max }$-dependence of the correlation function intercepts for different $\xi$-values. We can see that the width of the semi-inclusive correlation function increases with the increasing $n_{\max }$, while its intercept decreases at small $n_{\max }$, reaching a minimum at $n_{\max } \approx\langle n\rangle$, and then approaches the limiting value of 2 roughly as $\log n_{\max }$. The inclusive behavior is practically saturated at a moderate number of the included pions $n_{\max }=k\langle n\rangle$, where $k$ increases with the density parameter $\xi$ from about 3 at $\xi=0.89$ to about 5 at $\xi=0.99$. T4 Thus the neglect of the energy-momentum constraints in Eqs. (62) and (63) can be justified provided $\langle n\rangle \ll \sqrt{s} / m$, e.g., in the usual case of a logarithmic increase of the mean multiplicity with the c.m.s. energy $\sqrt{s}$. The situation can change in the case of very large and dense systems dominated by a soft condensate. Then the regime $\langle n\rangle \sim \sqrt{s}$ can settle, the energy-momentum constraints leading to the reduction of the maximal effective number of produced pions to $n_{\max } \sim\langle n\rangle$ and, as a result, to the suppression of the measured inclusive correlation function. Clearly, such an eventual suppression has nothing in common with the coherence effect.

\section{DISCUSSION AND CONCLUSION}

We have illustrated an approximate scaling of multiboson effects with the density parameters $\xi, \xi_{n}, \ldots$ (see, e.g., Figs. 2 and 5). It means that though our numerical results were obtained for typical AGS or SPS multiplicities of the order of tens or hundreds of pions, they are approximately valid also for higher multiplicities expected at RHIC or LHC energies.

The value of the density parameter $\xi$ can be estimated with the help of Eq. (48) relating the phase space density in the rare gas limit with the integrated correlation function. Thus using the usual Gaussian parameterization for the correlation function in the longitudinally co-moving system (LCMS):

$$
R\left(p_{1}, p_{2}\right)=1+\lambda \exp \left(-r_{x}^{2} q_{x}^{2}-r_{y}^{2} q_{y}^{2}-r_{z}^{2} q_{z}^{2}\right),
$$

where $x, y(\mathbf{y} \| \mathbf{z} \times \mathbf{p})$ and $z$ denote the outward, sideward and longitudinal directions respectively and parameterizing the single-particle spectra as

$$
N(\mathbf{p})=\frac{d n}{d y} \frac{\exp \left(-\left(m_{t}-m\right) / T\right)}{2 \pi T(T+m) m_{t} \cosh y}
$$

\footnotetext{
${ }^{14}$ The increase of the saturation point $n_{\max } /\langle n\rangle$ with the density is related with the increasing condensate contribution which, for the ideal BE gas, is characterized by very large multiplicity fluctuations.
} 
we arrive at the mean pion phase-space density

$$
\langle f\rangle_{\mathbf{p}}=\frac{\lambda \pi^{3 / 2}}{V} N(\mathbf{p}) \cosh y=\lambda \frac{\sqrt{\pi}}{2} \frac{\exp \left(-\left(m_{t}-m\right) / T\right)}{V T(T+m) m_{t}} \frac{d n}{d y},
$$

where $V=r_{x} r_{y} r_{z}$ is the LCMS interference volume. 15 For soft pions $\left(p_{t} \approx 0\right.$ and $\left.y \approx 0\right)$ at SPS energies this quantity is typically $\sim 0.2$. Since this value is sufficiently small, we can compare it with the model phase space density in the rare gas limit $\langle\tilde{f}\rangle_{\mathbf{p}=0} \approx \eta /\left(\sqrt{2} r_{0} \Delta\right)^{3}$ (see Eq. (47)) and get $\xi \approx 0.4-0.5$. For such values of the density parameter our calculations point to rather small multiboson effects in the ordinary events. These effects can show up however in the events containing sufficiently high density fluctuations. Particularly, the condensate effects could be seen in certain high multiplicity events (see, e.g., Fig. (7) in which the phase space volume or subvolume $\sim\left(r_{0} \Delta\right)^{3}$ does not follow the increasing multiplicity (as it presumably does in the ordinary events) and remains sufficiently small to guarantee a nonvanishing factor $\xi^{r_{0} \Delta}$ determining the condensate size (see Eqs. (66) and (71)).

Since at present energies the LCMS interference volume $V$ seems to scale with $d n / d y$, the freeze-out of the pions occurs on average at approximately constant phase space density (see Eq. (85)). In the rare gas regime, based on the density scaling one can then expect about the same relative size of the multiboson effects also at RHIC and LHC energies, up to a slight increase in $\xi$ due to the vanishing of the finite-size corrections with the increasing phase space volume of the emitting system. At the same time, the growing phase space volume will lead to suppression of the average condensate contribution, determined by the factor $\xi^{r_{0} \Delta}$.

Considering the multiboson effects in the low (BE gas) and the large (BE condensate) density limits, we have obtained simple analytical formulae accounting for the finite size of the phase space volume and allowing to follow the dependence of the mean multiplicities, single-boson spectra and two-boson correlation functions on the phase space density parameters. In principle, these formulae provide a possibility to identify multiboson effects among others. Particularly, the width of the low- $p_{t}$ enhancement due to the BE condensation decreases with the size of the system as $r_{0}^{-1 / 2}$ and this narrowing makes the observation of the effect easier.

The results of the considered simple model should not be taken, however, too literally since:

a) due to its static character, the model does not explicitly account for the experimental indications on a constant freeze-out phase space density and the related expansion of the emission volume. The qualitative application of our model to heavy ion collisions is however possible in the limited phase space regions. For example, the pions with a rapidity difference greater than about unity have to be considered as originating from different static sources; As discussed in Section IIB, the residual slow relative motion, if decoupled from other source characteristics, merely leads to a wider momentum dependence of the emission function in Eq. (42): $\Delta^{2} \rightarrow \Delta^{2}+\Delta_{0}^{2}$;

b) due to a mixture of different production processes (e.g., due to contribution of different impact parameters), the real multiplicity distribution and particle spectra will be rather weighted sums of those in Eqs. (29)-(31) calculated with different sets of the parameters $\eta^{i}, r_{0}^{i}, \Delta^{i}$. As a result, near the condensate limit, the multiplicity distribution can be wider than the BE one and the intercepts of the inclusive and fixed- $n$ correlation functions can differ from the respective single-process values of 2 and 1 ;

c) when estimating the freeze-out phase space density from the experimental data, the multiboson system is considered as a homogeneous medium. However, there can be large local density fluctuations - speckles which can give rise to noticeable multiboson effects even at a moderate value of the mean phase space density;

d) on the other hand, the multiboson effects can be somewhat suppressed due to a possible violation of the factorization assumption in Eq. (8) or due to the lack of the reflection symmetry of the emission volume. In latter case the functions $G_{n}\left(\mathbf{p}_{1}, \mathbf{p}_{2}\right)$ are no more real;

e) for identical charged pions, the BE effects are also suppressed due to the Coulomb repulsion. Since this repulsion is important only in a weakly populated region of very small relative momenta determined by the pair Bohr radius $a=387 \mathrm{fm}$, the suppression of the global BE weights $\omega_{n}$ is rather small. For example, for $\omega_{2}$ this suppression, being about $\left(a r_{0} \Delta^{2}\right)^{-1}$, is usually less than one per mill. The Coulomb distortion of the global multiboson effects is therefore negligible in the rare gas limit. Nevertheless, since the Coulomb repulsion destroys the formation of the condensates made up from positive and negative pions in the disjoint phase space regions, it can lead to noticeable differences between charged and neutral pions in dense systems. Particularly, we can expect a decrease of the charge-to-neutral multiplicity ratio with the increasing phase space density.

Because of large numbers of positive and negative pions produced in heavy ion collisions, one could also raise a question about importance of the Coulomb screening effects violating standard two-body treatment of the correlations

\footnotetext{
${ }^{15}$ A better estimate may require the substitution [9] $\lambda \rightarrow \lambda^{1 / 2}$ in Eq. (85).
} 
in the low density limit. There are however arguments showing that the screening will be of minor importance even at LHC [15] (see also [27]). Note that in the scenario with a constant phase-space density the corresponding Debye radius

$$
r_{D}=\left[4 \pi\left(\rho_{+}+\rho_{-}\right) e^{2} / T\right]^{-1 / 2}
$$

where $e^{2}=1 / 137$ and $\rho_{+}+\rho_{-}$is the total density of charged pions in the configuration space, will be also constant, up to a weak energy dependence due to the temperature T. Assuming that pions with a rapidity difference greater than unity come from spatially disjoint regions of phase-space, we can put 15]

$$
\rho_{+}=\left(\frac{\lambda}{(2 \pi)^{3}}\right)^{1 / 2} \frac{d n_{+} / d y}{V}=\frac{\sqrt{2}}{\sqrt{\lambda} \pi^{2}} T^{3}\left\langle f_{+}\right\rangle_{y}
$$

and obtain $r_{D} \approx 15 \mathrm{fm}$ at $\left\langle f_{+}\right\rangle_{y} \approx 0.1$ and $T \approx 200 \mathrm{MeV} / \mathrm{c}\left(r_{D} \sim 1 / T\right)$. Thus at LHC energies we can expect the characteristic distances between the pion production points comparable or larger than the screening radius $r_{D}$ leading to a suppression of the usual two-particle Coulomb effects. In fact, two charged pions produced at a distance $r^{*}>r_{D}$ start to feel their Coulomb field only after some time when the density decreases to a value corresponding to Debye radius larger than $r^{*}$. During this time the vector of the relative distance between the pion emission points increases by $[15$

$$
\Delta \mathbf{r}^{*} \sim \frac{\mathbf{k}^{*}}{(m T)^{1 / 2}} V^{1 / 3}\left[\left(\frac{r^{*}}{r_{D}}\right)^{2 / 3}-1\right] .
$$

Substituting $\mathbf{r}^{*}$ by $\mathbf{r}^{*}+\Delta \mathbf{r}^{*}$ in the argument of the Coulomb wave function, we can see however that the suppression of the Coulomb effect can be substantial only in the region of large relative momenta $k^{*}>(m T)^{1 / 2}$ where the correlations due to QS and FSI are already negligible (see, e.g., [19,28).

Finally, to make easier the understanding of our results in context of other papers and, for the reader's convenience, we compare our results (including those in [15]) with the results of refs. [5, 6, 16, 17] and the recent papers [29 34,36 39] which appeared either after the present work was basically completed or during the process of its evaluation.

In the pioneering papers [5],6], the analytically solvable model discussed in Section [V] was introduced. A simple algorithm was given allowing to calculate BE modified multiplicity distribution, single- and two-boson spectra in terms of the quantities $C_{n}^{\text {Pratt }}=\widetilde{g}_{n} / n, G_{n}^{\text {Pratt }}\left(\mathbf{p}_{1}, \mathbf{p}_{2}\right)=\widetilde{G}_{n}\left(\mathbf{p}_{1}, \mathbf{p}_{2}\right)$. The details of the calculation of the $G_{n}$-functions and of the two-boson spectra were not given in [5,6]. They can be found in [15] [18] (see also [29,30]). In refs. [29] this technique was extended to the spectra and correlations of three or more pions. It was shown that, in the inclusive case, the old formalism, not accounting for the multiboson effects, can be recovered by a redefinition of the Wigner-like density which then becomes more narrow both in momentum and configuration space.

Regarding the choice of the Wigner-like density $D(p, x)$, in the original papers [5, 6] and in [16], it was slightly different from that in Eq. (42), corresponding to the substitution $\exp \left(-\mathbf{q}^{2} / 2 \Delta^{2}\right) \rightarrow \exp \left(-p_{0} / T\right)$, where $T=\Delta^{2} / m$. This choice is, in fact, in contradiction with the uncertainty principle, the latter requiring an energy independent density in the considered case of a fixed emission time $t=0$ (see Eq. (20)). (For the same reason, the freedom in the choice of the parameters $r_{0}, \Delta$ is limited by the inequality $r_{0} \Delta \geq 1 / 2$, the equality corresponding to the zero distance between the emitter centers.) The incorrect choice of the Wigner-like density in [5.66.16] can be cured by the substitution $r_{0}^{2} \rightarrow r_{0}^{2}-(2 \Delta)^{-2}$. To recover the recurrence relations in Eqs. (50), besides this substitution, one has to take into account that the width and the slope parameters used e.g. in [16] are related to ours as: $R_{0}^{2}=2 r_{0}^{2}, p_{0}^{2}=\Delta^{2}, a_{n}=b_{n}^{+}+b_{n}^{-}, g_{n}=2\left(b_{n}^{+}-b_{n}^{-}\right)$and that a factor of $R_{0}^{2}$ is missing in Eq. (66) for $g_{n+1}$ in [16].

The analytical solution for the $G_{n}$-functions has been found in [17,30,31]. In [17 the same form of the Wigner-like density as in Eq. (42) was used with the parameters $R_{\text {eff }}^{2}=r_{0}^{2}$ and $\sigma_{T}^{2}=2 \Delta^{2}$. However, since the analytical solution was derived based on the recurrence relations of ref. [16], it has also to be cured by the substitution $r_{0}^{2} \rightarrow r_{0}^{2}-(2 \Delta)^{-2}$. Particularly, after this substitution, Eq. (200) in the preprint version of [17] for the critical multiplicity $\eta_{c}$ (coinciding with Eq. (9) in [5] corrected for the misprints [17]) then reduces to the simple result [15]: $\eta_{c}=\beta$. In [30], the analytical solution given in Eqs. (2.14) corresponds to the emission function in Eq. (42) with the parameters $r_{0}^{2}=R^{2} / 2+\left(2 p_{0}\right)^{-2}, \Delta^{2}=p_{0}^{2} \cdot 16$ Requiring a matrix algebra, this solution is however less transparent compared

\footnotetext{
${ }^{16}$ Eqs. (2.14b) and (2.14d) have to be corrected for the misprints by the substitutions: $1 / 4 \rightarrow(1+c / 2) / 4$ and $(1+c) \rightarrow(1+c / 2)$ respectively. Note that the other solution given in Eqs. (4.3), (4.4) of ref. [30] presumably contains an error since, corresponding to a Gaussian factorizable model, it does not reduce to the general solution in Eqs. (49 51) (particularly, it shows no condensate behavior at high densities).
} 
with that in [17]. In [31], the analytical solution was obtained using the technique of the density matrix in the 1-dimensional momentum space. Generalizing this solution to three dimensions (by substituting the normalization factors $\lambda_{0}^{n} /\left[1-\left(1-\lambda_{0}\right)^{n}\right]$ and $\left(2 \pi \hat{\Delta}_{n}^{2}\right)^{-1 / 2}$ by their cubes) and relating the notation of ref. 31] to ours: $\hat{\Delta}_{n}^{-2}=8 b_{n}^{+}$, $\hat{R}_{n}^{2}=2 b_{n}^{-}, \omega_{n}^{B Z}=\left(1-\lambda_{0}\right)^{n}=\rho^{n}, L\left(p_{1}, p_{2}\right)=\widetilde{G}_{n}\left(p_{1}, p_{2}\right)$, one can see that it then coincides with the solution in Eqs. (51).

As for the analytical approximations of the mean multiplicity, single- and two-boson inclusive spectra, similar results as in ref. [15] have been obtained in 17] in both the low and high density regimes. The behavior of the single-boson spectrum in the low density limit was also studied numerically in [32] - a linear increase of the relative correction with the phase space density was found (in agreement with Eq. (71) at small $\widetilde{\xi}_{\mathbf{p}}$ ). The condensate behavior at very high densities was also obtained in [31]. A comment requires the effective radius squared, estimated as [31] $r_{\text {eff }}^{2}=\left(2\left\langle\mathbf{q}^{2}\right\rangle\right)^{-1}$, where the averaging over the correlation term is assumed. With the increasing mean multiplicity, $r_{\text {eff }}^{2}$ decreases from $r_{0}^{2}$ to $r_{0}^{2} /\left(2 r_{0} \Delta\right)$. Note, however, that at high densities, $r_{\text {eff }}^{2}$ has little to do with the interferometry radius squared. The latter accounts also for the change of the single-boson spectrum and, with the increasing density, decreases from $r_{0}^{2}-(2 \Delta)^{-2}$ to 0 .

A special comment requires the normalization of the correlation functions. There are two popular definitions of the two-particle correlation function, both representing a ratio of the two-particle spectrum to the product of the singleparticle ones, but differing in the normalization. In case (I) the spectra are normalized to 1 while in case (II) - to the numbers of single- and two-particle counts. This definitions correspond to Eq. (35) or (36) with (I) $c_{n}=n /(n-1)$ or $c=\langle n\rangle^{2} /\langle n(n-1)\rangle$ and (II) $c_{n}=c=1$. In the inclusive case, based on the thermal-type models (see the comment after Eq. (63)), the second choice was advocated [33,34]. Recall that the first choice (I) would be preferable in case of a fixed pion multiplicity provided a large phase space volume (see discussion after Eq. (59)). Generally, however, there is no well-defined normalization and, for a reliable comparison with the experimental correlation functions, a free normalization parameter, depending on the production mechanism (not necessarily a single thermal one) and experimental conditions, has to be introduced [15].

The role of the normalization was misunderstood in 116,17, where the decrease of the intercept of the inclusive correlation function (I) with the increasing phase space density, obtained in the factorizable Gaussian model, was incorrectly interpreted as a coherent laser behavior. Recall that pions in a coherent state would have a narrow Poisson multiplicity distribution, while the BE condensate is characterized by very wide multiplicity fluctuations. Clearly, the coherent pion production requires a special mechanism (not present in the model), like that leading to possible formation of the disoriented chiral condensate - DCC (for a review, see [35]). Possibilities of experimental investigations of BE condensate and DCC phenomena have been recently discussed in [36]. A discussion of statistical physics aspects of the multiboson effects can be found in [5, 17, 30, 34, 37, 39].

In conclusion we summarize the results.

- The influence of the multiboson effects on boson multiplicities, single-boson spectra and two-boson correlations, including an approximate scaling behavior of some of their characteristics with the phase space density $(e . g .,\langle n\rangle / \eta$ vs $\xi$ or $N_{n}(0) / \beta_{n}$ vs $\left.\xi_{n}\right)$, has been demonstrated using the analytically solvable Gaussian model.

- The approximate analytical formulae are given allowing to follow the dependence of these quantities on the phase space density parameters thus providing a possibility for the identification of the multiboson effects among others.

- The meaning and the applicability conditions of the model factorization assumption are clarified using the physically transparent Kopylov-Podgoretsky ansatz of classical one-particle sources. For heavy ion collisions, the factorizatiom assumption is expected to be valid in case of an impact parameter selection.

- The lowest order cumulant approximation, suggested for a practical account of multiboson effects in realistic transport code simulations [18], has been shown to be reasonable at moderate densities indicated by the experimental data.

- At high densities, the spectra are mainly determined by the universal condensate term $P_{c}(\mathbf{p})\left(e . g ., N_{n}^{(1)}(\mathbf{p}) \rightarrow\right.$ $n P_{c}(\mathbf{p})$ and $\left.N_{n}^{(2)}\left(\mathbf{p}_{1}, \mathbf{p}_{2}\right) \rightarrow n(n-1) P_{c}\left(\mathbf{p}_{1}\right) P_{c}\left(\mathbf{p}_{2}\right)\right)$ and the initially narrow Poisson multiplicity distribution approaches a wide BE one: $\langle n(n-1)\rangle \rightarrow 2\langle n\rangle^{2}$. As a result, the intercepts of the inclusive and fixed- $n$ correlation functions (properly normalized to 1 at large $|q|$ ) approach 2 and 1 , respectively and their low- $\mathbf{q}^{2}$ slopes rapidly vanish with increasing density; the corresponding increase of the apparent correlation function width is however rather slow logarithmic in the density.

- It is found that, even near the condensate regime, the inclusive characteristics saturate at rather moderate multiplicities of some multiples of $\langle n\rangle$ thus justifying the neglect of energy-momentum constraints in the considered analytical model. The latter are however important near a multipion threshold, particularly making impossible the production of a very cold BE condensate.

- Though spectacular multiboson effects are hardly to be expected in typical events of heavy ion collisions in present and perhaps also in future heavy ion experiments, they can clearly show up in certain classes of events containing sufficiently high density fluctuations. 


\section{ACKNOWLEDGMENTS}

This work was supported in part by GA Czech Republic, Grant No. 202/98/1283, by Russian Foundation of Fundamental Investigations, Grant No. 97-02-16699, by Ukrainian State Fund for the Fundemental Research, Contract No. 2.5.1/057 and by Ukrainian-Hungarian Grant No. 2M/125-199.

[1] M.I. Podgoretsky, Fiz. Elem. Chast. Atom. Yad. 20, 628 (1989) (Sov. J. Part. Nucl. 20, 266 (1989)).

[2] M.I. Podgoretsky, JINR Report P2-85-240 (1985).

[3] W. Zajc, Phys. Rev. D 35, 3396 (1987).

[4] W.N. Zhang, Y.M. Liu, S. Wang, Q.J. Liu, J. Jiang, D. Keane, Y. Shao, S.Y. Chu, S.Y. Fung, Phys. Rev. C 47, 795 (1993).

[5] S. Pratt, Phys. Lett. B 301, 159 (1993).

[6] S. Pratt, Phys. Rev. C 50, 469 (1994);

S. Pratt, V. Zelevinsky, Phys. Rev. Lett. 72, 816 (1994).

[7] G.F. Bertsch, Phys. Rev. Lett. 72, 2349 (1994).

[8] Yu.M. Sinyukov, B. Lorstad, Z. Phys. C 61, 587 (1994).

[9] J. Barrette et al. (E877 Collaboration), Phys. Rev. Lett. 78, 2916 (1997).

[10] B. Jacak, Proc. Int. Workshop on Multiparticle Correlations and Nuclear Reactions, CORINNE II, Nantes, France, 1994 (ed. J. Aichelin and D. Ardouin, World Scientific, 1994) p. 3.

[11] M. Gazdzicki, Nucl. Phys. A590, 197c (1995).

[12] P.V. Degtyarenko, E.A. Doroshkevich, Yu.V. Efremenko, V.B. Gavrilov, M.V. Kossov, G.A. Leksin, A.V. Stavinsky, A.V. Vlassov, Z. Phys. A 350, 263 (1995).

[13] S.V.Akkelin, Yu.M. Sinyukov, Phys. Lett. 356, 525 (1995).

[14] Yu.M. Sinyukov, B. Lorstad, V.A. Averchenkov, Z. Phys. C 49, 417 (1991).

[15] B. Erazmus, R. Lednicky, V. Lyuboshitz, L. Martin, K. Mikhailov, J. Pluta, Yu. Sinyukov, A. Stavinsky, K. Werner, ALICE Internal Note 95-43, Geneva, 1995;

R. Lednicky, Proc. Int. Workshop Demon in Warsaw, Warsaw, Poland, 1997, in: Nukleonika 43, 345 (1998); Proc. Int. Conf. Hadron Structure '98, Stara Lesna, Slovakia, 1998, p. 293.

[16] W.Q. Chao, C.S. Gao, Q.H. Zhang, J. Phys. G: Nucl. Phys. 21, 847 (1995);

Q.H. Zhang, W.Q. Chao, C.S. Gao, Phys. Rev. C 52, 2064 (1995).

[17] T. Csorgo, J. Zimanyi, Phys. Rev. Lett. 80, 916 (1998);

J. Zimanyi, T. Csorgo, CU-TP-818/1997, hep-ph/9705432 v2.

[18] N.S. Amelin, R. Lednicky, Heavy Ion Physics 4, 241 (1996); SUBATECH 95-08, Nantes 1995.

[19] R. Lednicky, V.L. Lyuboshitz, Proc. Int. Workshop on Particle Correlations and Interferometry in Nuclear Collisions, CORINNE 90, Nantes, France, 1990 (ed. D. Ardouin, World Scientific, 1990) p. 42; JINR report P2-546-92 (1992); Heavy Ion Physics 3, 93 (1996).

[20] S. Pratt, Phys. Rev. Lett. 53, 1219 (1984); Phys. Rev. D 33, 1314 (1986).

[21] R. Lednicky, V.L. Lyuboshitz, M.I. Podgoretsky, Yad. Fiz. 38, 251 (198;3) (Sov. J. Nucl. Phys. 38, 147 (1983)); V.L. Lyuboshitz, Yad. Fiz. 53, 823 (1991) (Sov. J. Nucl. Phys. 53, 514 (1991)).

[22] I.V. Andreev, R.M. Weiner, Phys. Lett. B 253, 416 (1991);

Yu.M. Sinyukov, A.Yu. Tolstykh, ITP-92-5E, Kiev, 1992; Z. Phys. C 61, 593 (1994).

[23] J. D. Bjorken, Phys. Rev. D 27, 1327 (1983).

[24] L.D. Landau, E.M. Lifshitz, Statistical Physics: 2-nd Ed., Sect. 59, Pergamon Press, Oxford, 1969.

[25] V.A. Karmanov, A.E. Kudryavtsev, Proc. Symp. on Nucleon-Nucleon and Hadron-Nucleus Interactions at Intermediate Energies, Leningrad 1986, p. 558; ITEP-88-1983; Phys. of Atomic Nuclei 62, 895 (1999).

[26] Proc. of "Quark Matter 93", Nucl. Phys. A566 (1994).

[27] D.V. Anchishkin, W.A. Zajc, G.M. Zinovjev, Ukrainian J. Phys. 41, 363 (1996).

[28] R. Lednicky, V.L. Lyuboshitz, Yad. Fiz. 35, 1316 (1982) (Sov. J. Nucl. Phys. 35, 770 (1982)).

[29] Q.H. Zhang, Phys. Letters B 406, 366 (1997); Nucl. Phys. A634, 190 (1998); Phys. Rev. C 58, R18 (1998).

[30] U.A. Wiedemann, Phys. Rev. C 57, 3324 (1998).

[31] A. Bialas, K. Zalewski, Phys. Letters B 436, 153 (1998).

[32] R.L. Ray, Phys. Rev. C 57, 2523 (1998).

[33] D. Miskowiec, S. Voloshin, nucl-ex/9704006.

[34] Q.H. Zhang, P. Scotto, U. Heinz, Phys. Rev. C 58, 3757 (1998).

[35] J.D. Bjorken, Acta Phys. Polonica B 28, 2773 (1997). 
[36] S.V. Akkelin, Yu.M. Sinyukov, nucl-th/9909009; Proc. Int. Conf. Quark Matter '99, Torino, 1999;

A. Bialas, K. Zalewski, Phys. Rev. D 59, 975029 (1999).

[37] Yu.M. Sinyukov, S.V. Akkelin, R. Lednicky, nucl-th/9909015; Proc. 8-th Int. Workshop on Multiparticle Production: Correlations and Fluctuations '98, Matrahaza, Hungary, 1998, p. 66.

[38] A.Z. Mekjian, B.R. Schlei, D. Strottman, Phys. Rev. C 58, 3627 (1998).

[39] A. Bialas, K. Zalewski, Acta Phys. Slovaca 49, 145 (1999).
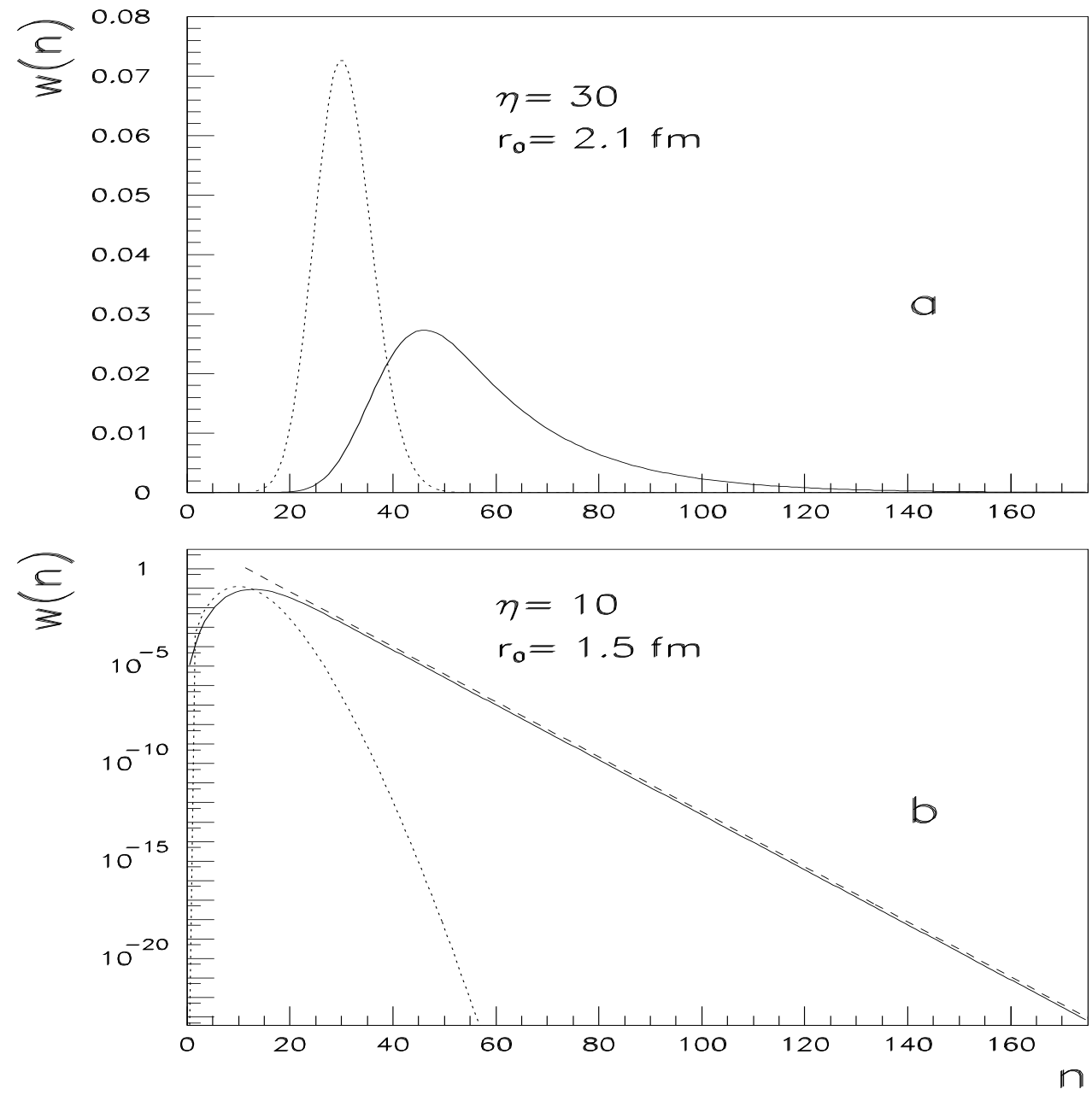

FIG. 1. The multiplicity distribution of neutral pions for a) $\Delta=0.25 \mathrm{GeV} / \mathrm{c}, r_{0}=2.1 \mathrm{fm}, \eta=30$ and b) $\Delta=0.25 \mathrm{GeV} / \mathrm{c}$, $r_{0}=1.5 \mathrm{fm}, \eta=10$, where $\eta$ is the mean multiplicity of the initial Poissonian distributions (dotted curves). The dashed line corresponds to Eq. (64). 

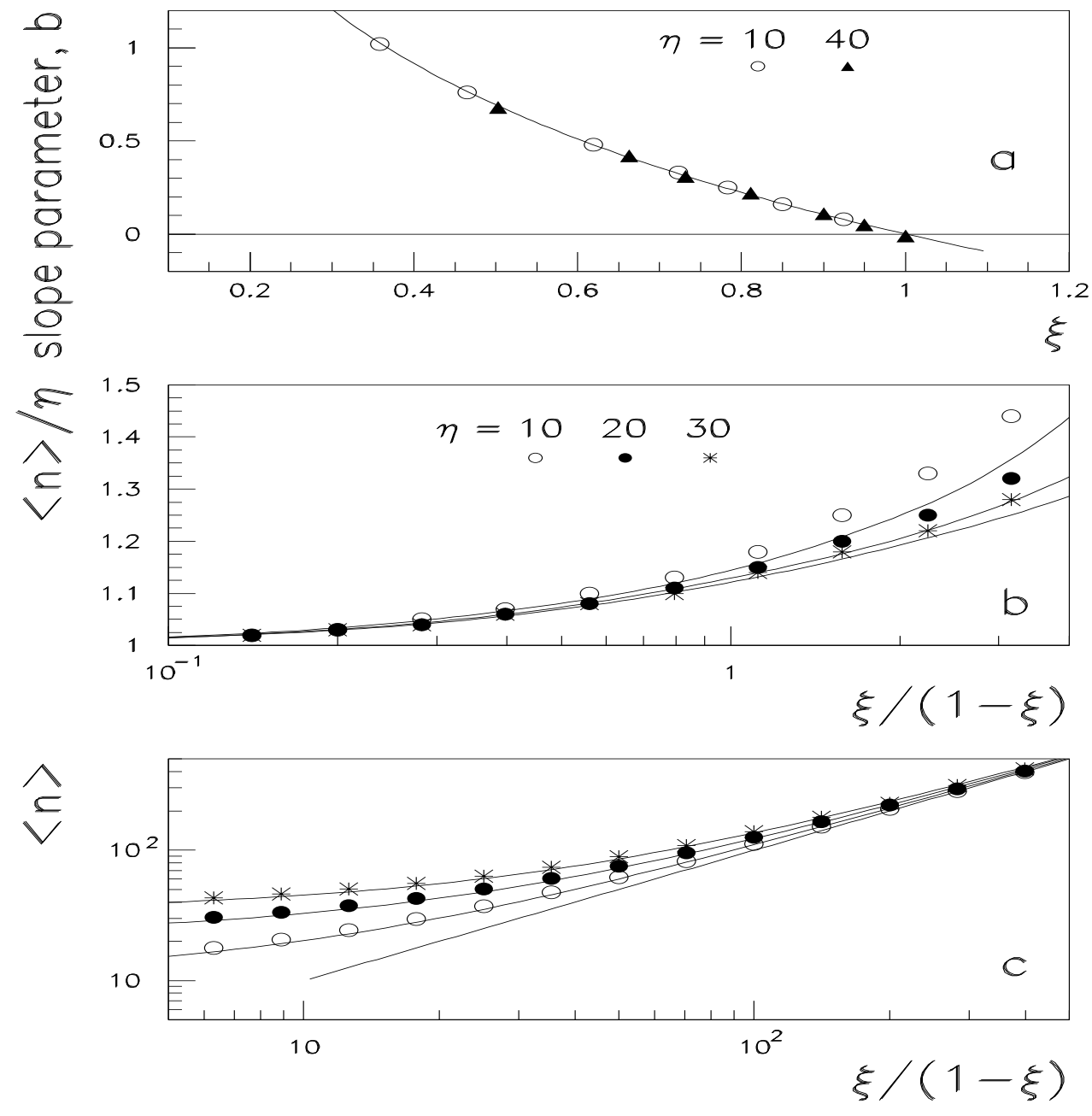

FIG. 2. The slope parameter $b$ of the exponential tail $c \cdot \exp (-b \cdot n)$ of the multiplicity distribution (a), the ratio of the mean multiplicity to the initial Poissonian one (b) and the mean multiplicity (c) as functions of the density parameters $\xi=\eta / \beta$ and $\xi /(1-\xi) ; \Delta=0.25 \mathrm{GeV} / \mathrm{c}$. The curve in (a): $b=-\ln \xi$, the curves in (b), (c) are calculated according to the tailing approximation in Eq. (66), the line in $(\mathrm{c}):\langle n\rangle=\xi /(1-\xi) \equiv \nu$. 


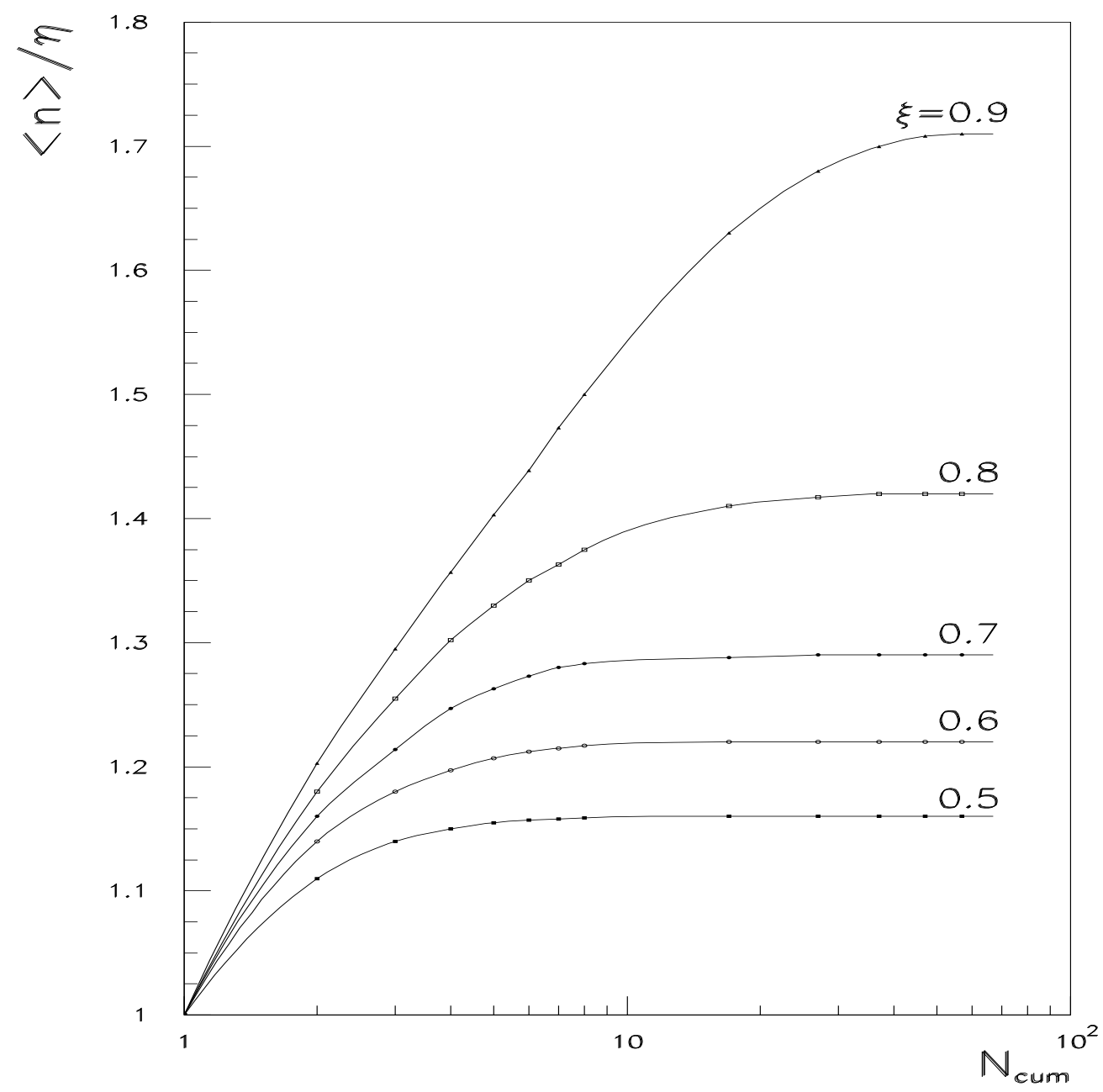

FIG. 3. The ratio of the mean multiplicity to the initial Poissonian one as a function of the number of the contributing cumulants for different values of the density parameter $\xi$. 

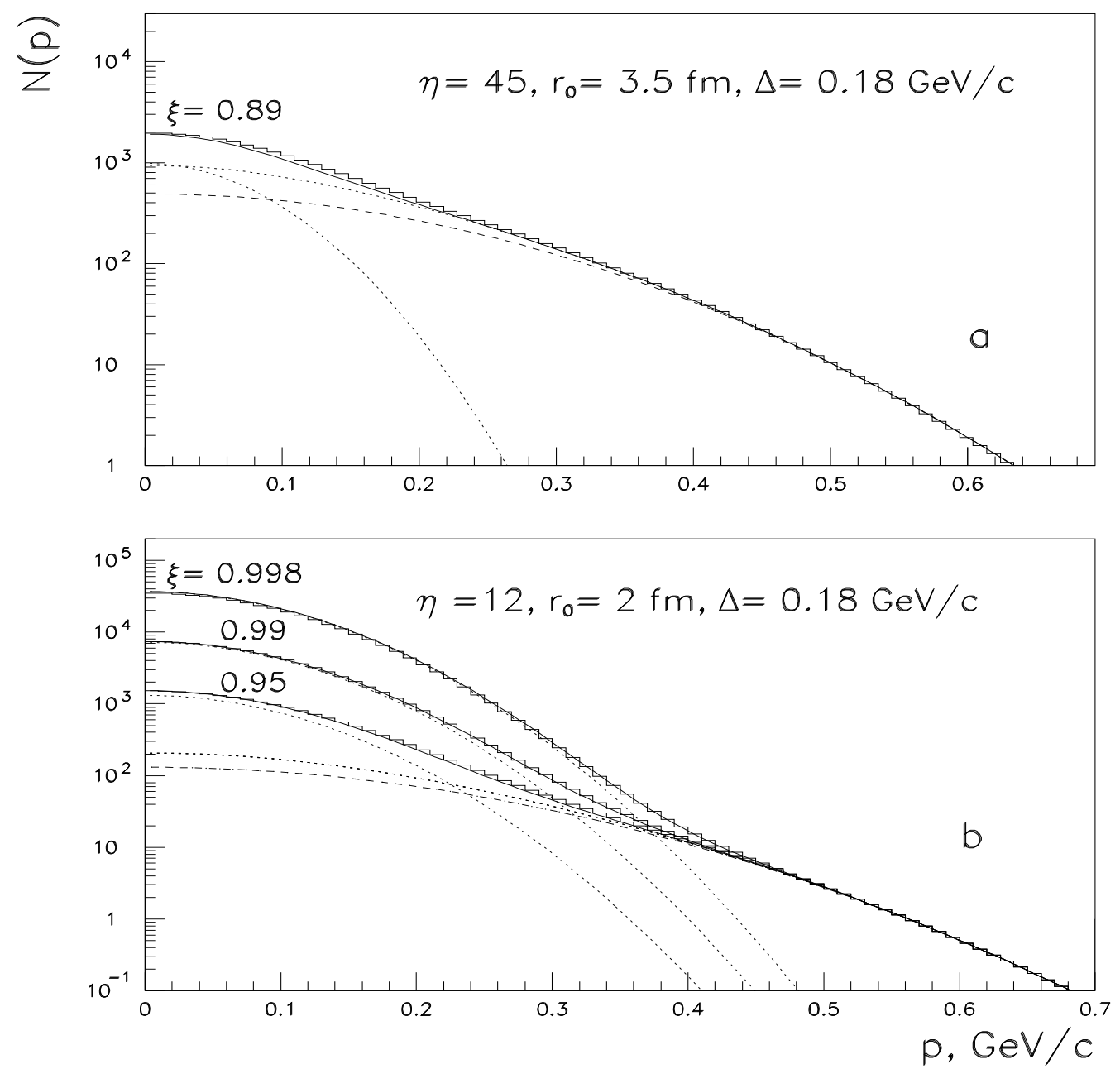

FIG. 4. The inclusive single-particle spectra corresponding to the density parameters a) $\xi=0.89$ and b) $\xi=0.95,0.99$ and 0.998 (the radius $r_{0}$ slightly varies near $2 \mathrm{fm}$ ); the corresponding mean multiplicities are a) 64.3 and b) $33.5,113.7$ and 433.8 . The histograms represent the exact result, the full curves are calculated according to the tailing approximation (71), the dotted ones represent the contributions of the two (BE gas and BE condensate) terms in Eq. (71) and the dashed curves correspond to the rare gas limit $\eta P(\mathbf{p})$. 

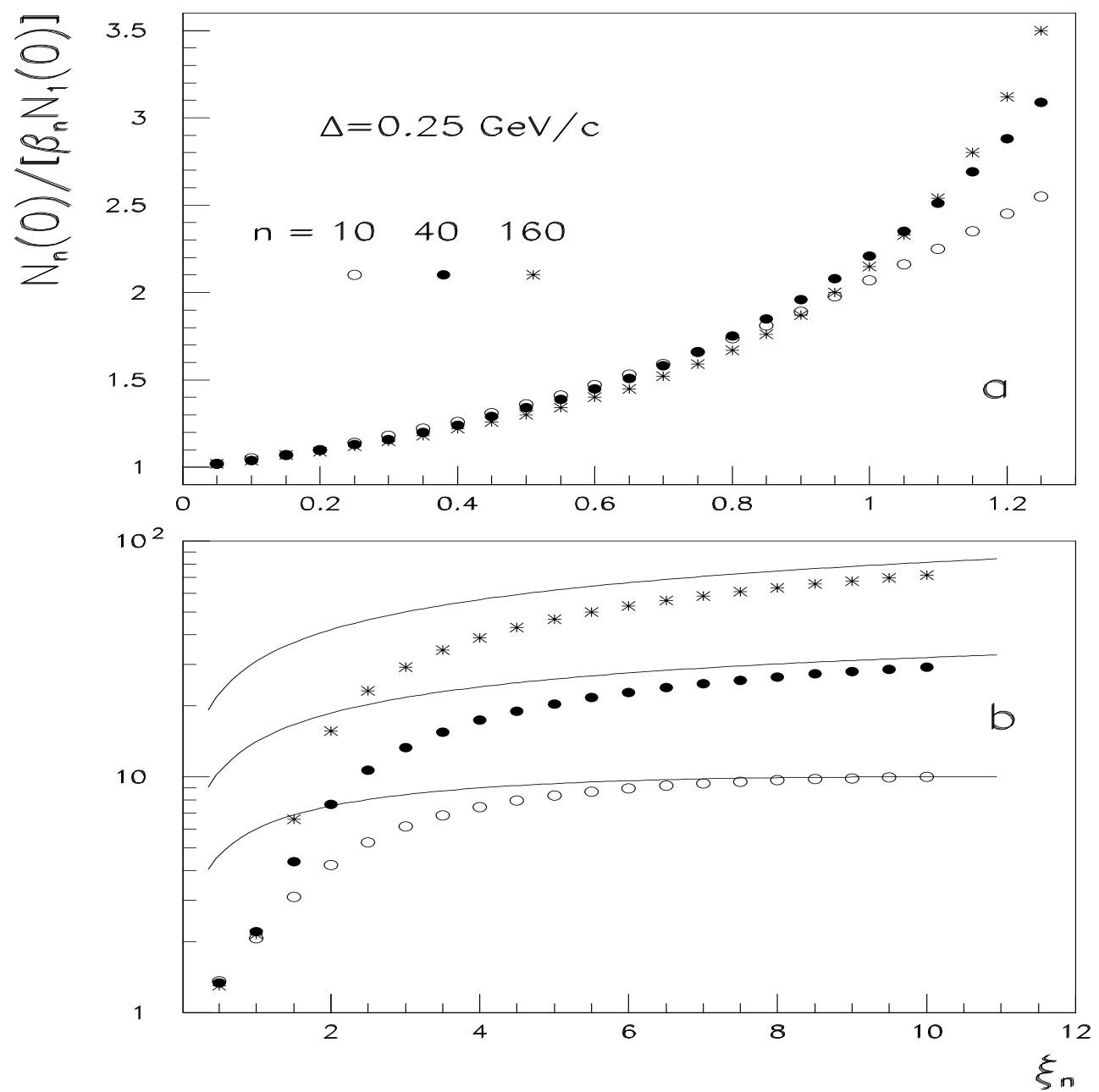

FIG. 5. The ratios of the BE affected single-particle spectrum to the dominant large-p contribution $\beta_{n} N_{1}(\mathbf{p})$, $\beta_{n}=n \omega_{n-1} / \omega_{n}$, calculated at $\mathbf{p}=0$ as functions of the density parameter $\xi_{n}=n / \beta$. The curves represent the large- $\xi_{n}$ limit: $\left(2 r_{0} \Delta\right)^{3 / 2} \xi_{n} \equiv\left[2\left(n / \xi_{n}\right)^{1 / 3}-1\right]^{3 / 2} \xi_{n}$. 


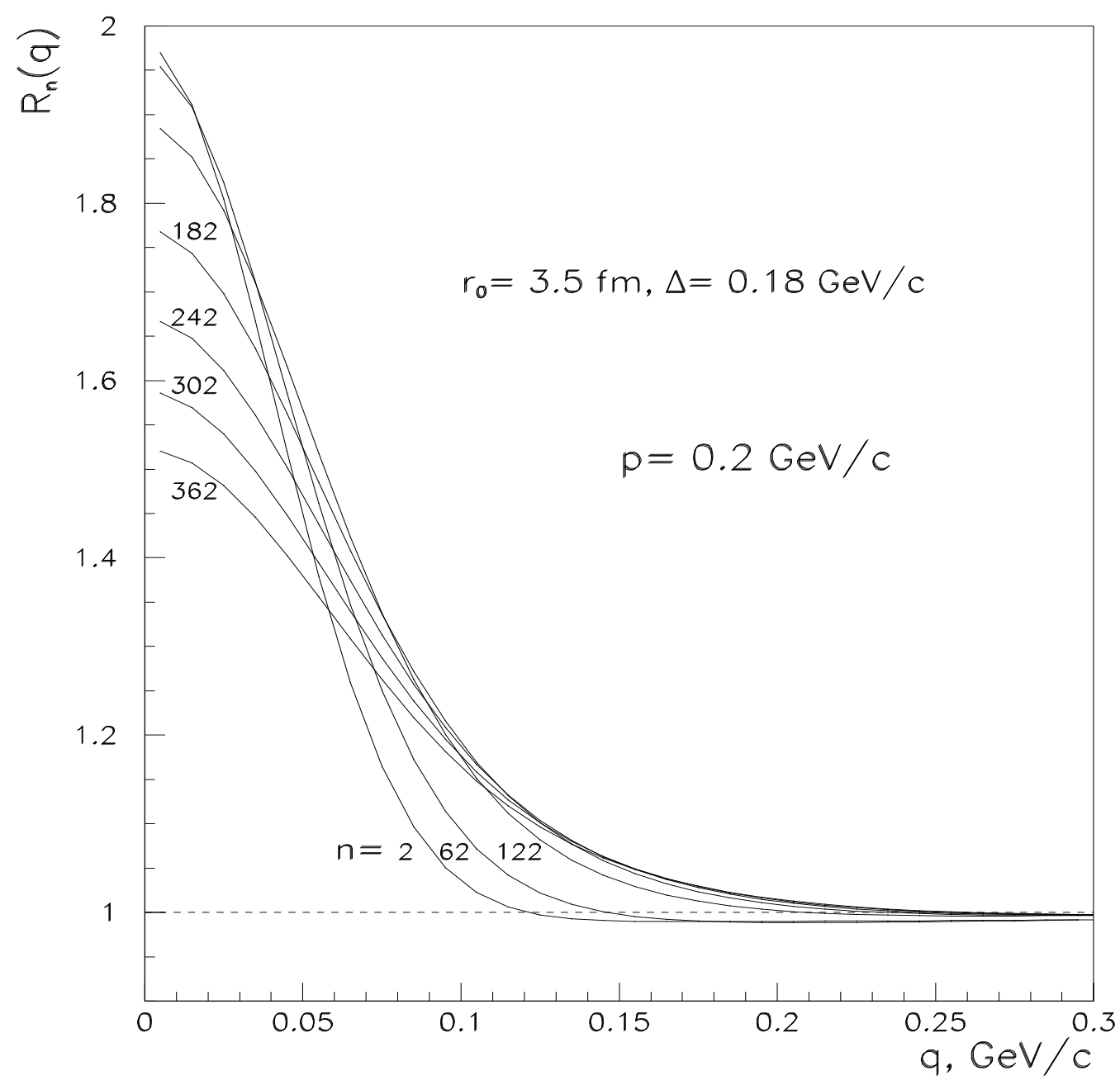

FIG. 6. The two-pion correlation functions for the multiplicities increasing from $n=2$ to 362 with a step of 60 (the corresponding density parameter $\xi_{n}$ ranging from 0.04 to 7.2 with a step of 1.2 ). The higher is the multiplicity the lower is the intercept of the correlation function and the larger is its width. 

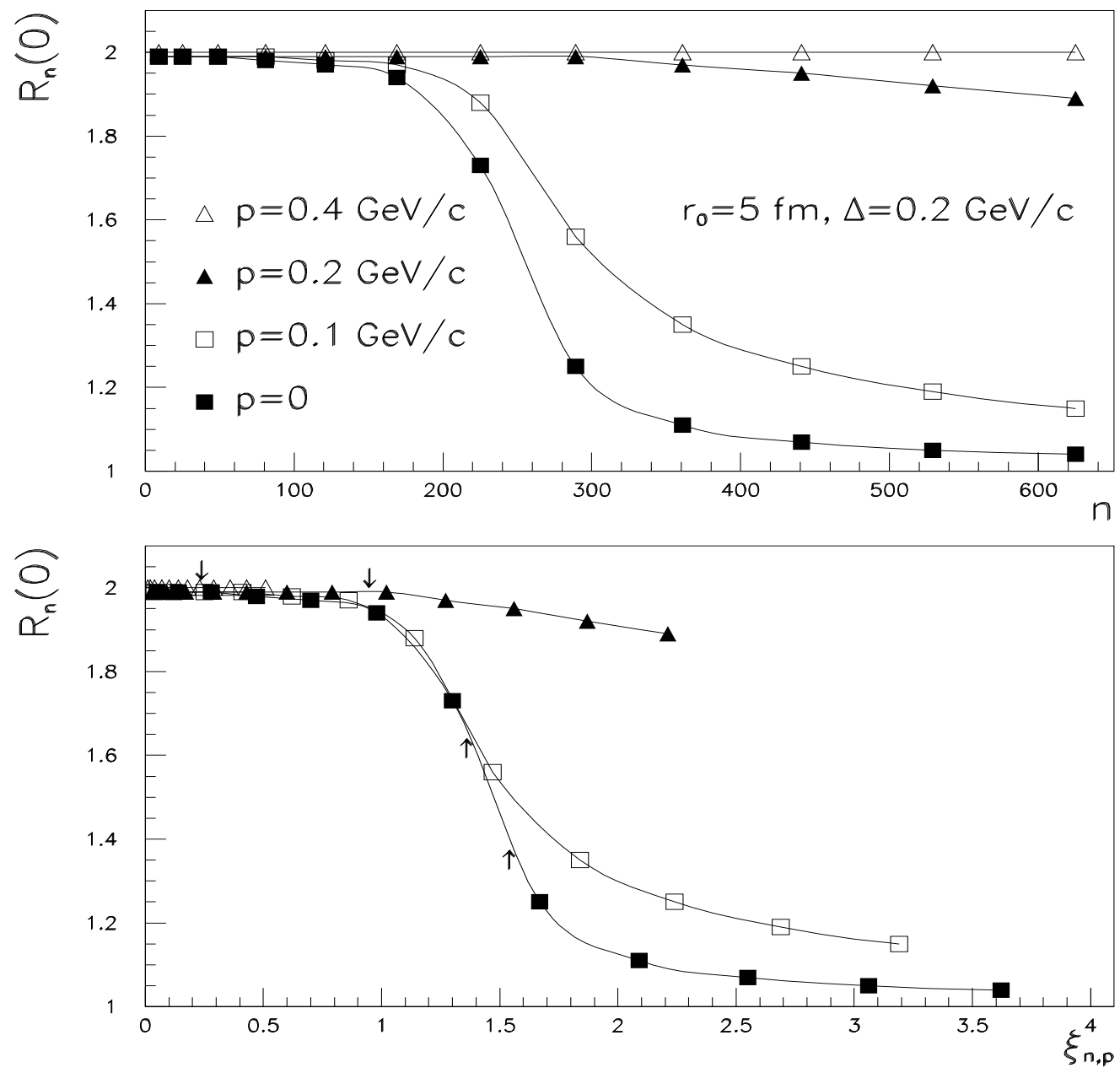

FIG. 7. The intercept of the two-pion correlation functions as a function of the multiplicity $n$ and the density parameter $\xi_{n, \mathbf{p}}$ for several values $p=0,0.1,0.2$ and $0.4 \mathrm{GeV} / \mathrm{c}$ of the mean momentum of the two pions. The arrows on the interpolating curves indicate the intercept values corresponding to $\xi_{n}=3 \xi=1.5(n \approx 3\langle n\rangle)$. 


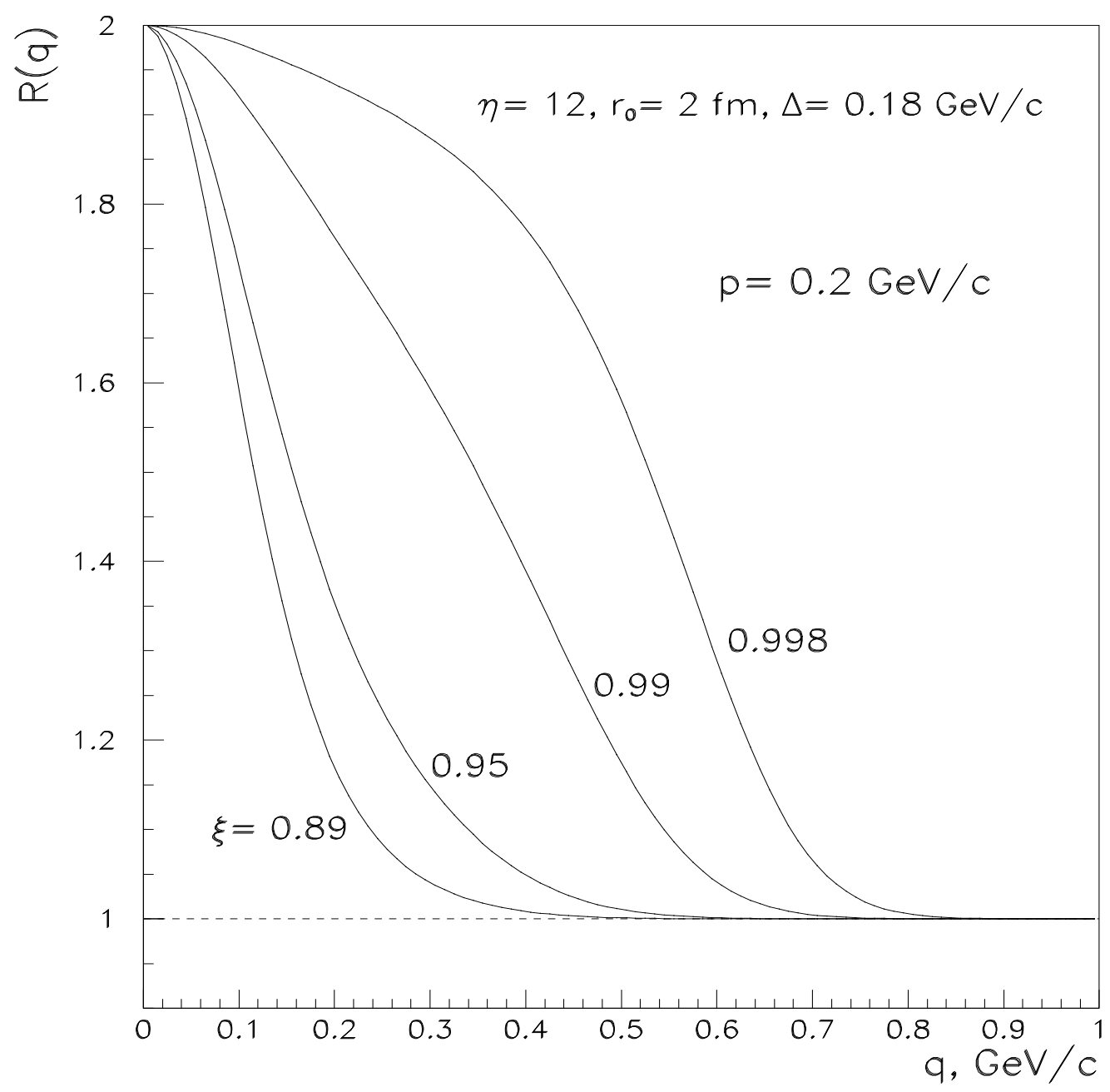

FIG. 8. The inclusive two-pion correlation functions demonstrating the increase of the correlation width with the increasing density parameter $\xi$. The different $\xi$-values are achieved by slight variations of the radius $r_{0}$ around $2 \mathrm{fm}$. 


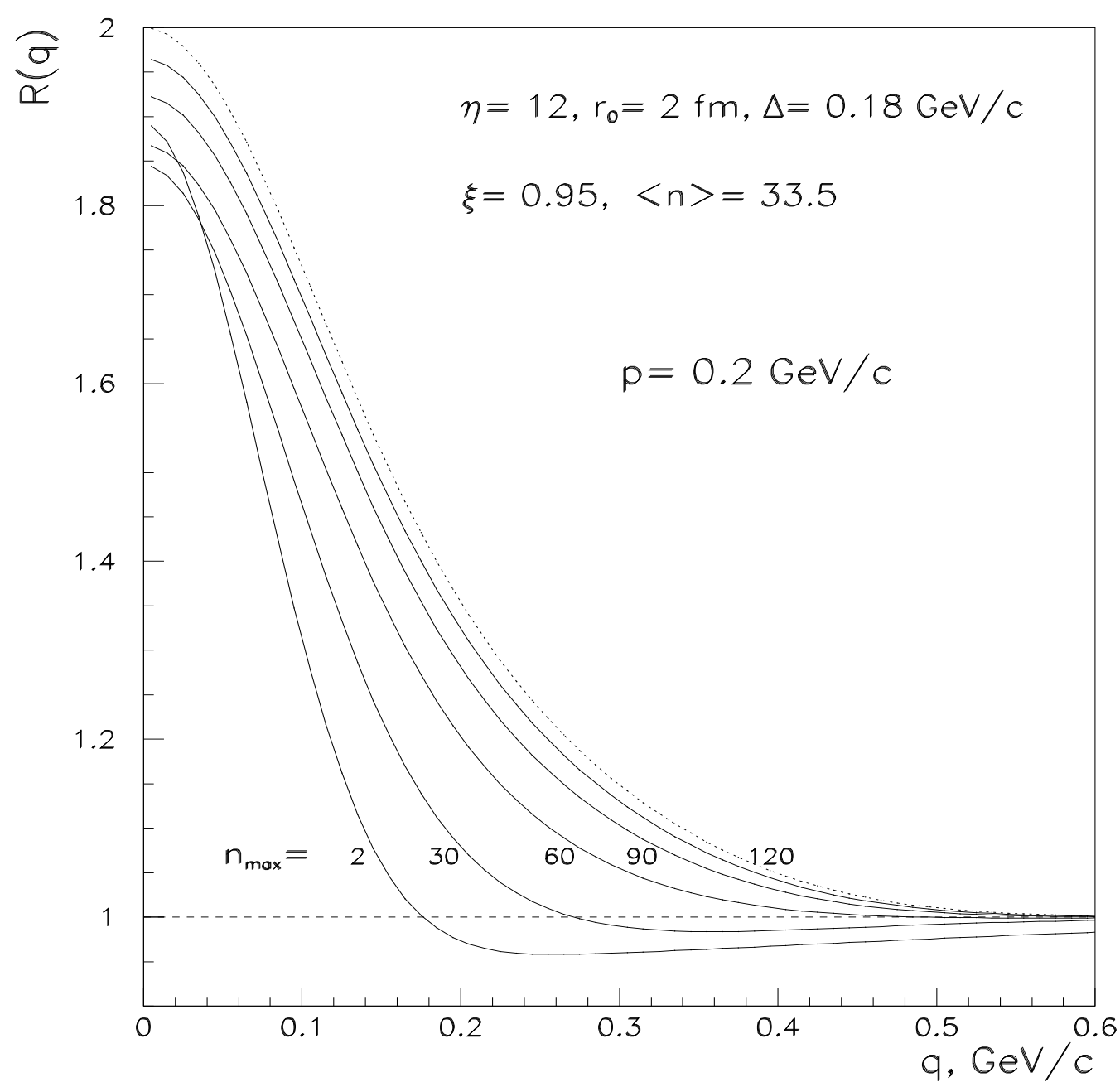

FIG. 9. The semi-inclusive correlation functions including the pion multiplicities from 0 to $n_{\max }$ for different values of $n_{\max }$. The dotted curve is the inclusive $\left(n_{\max } \rightarrow \infty\right)$ correlation function. The conditions are the same as in Fig. 8 for the density parameter $\xi=0.95$. 


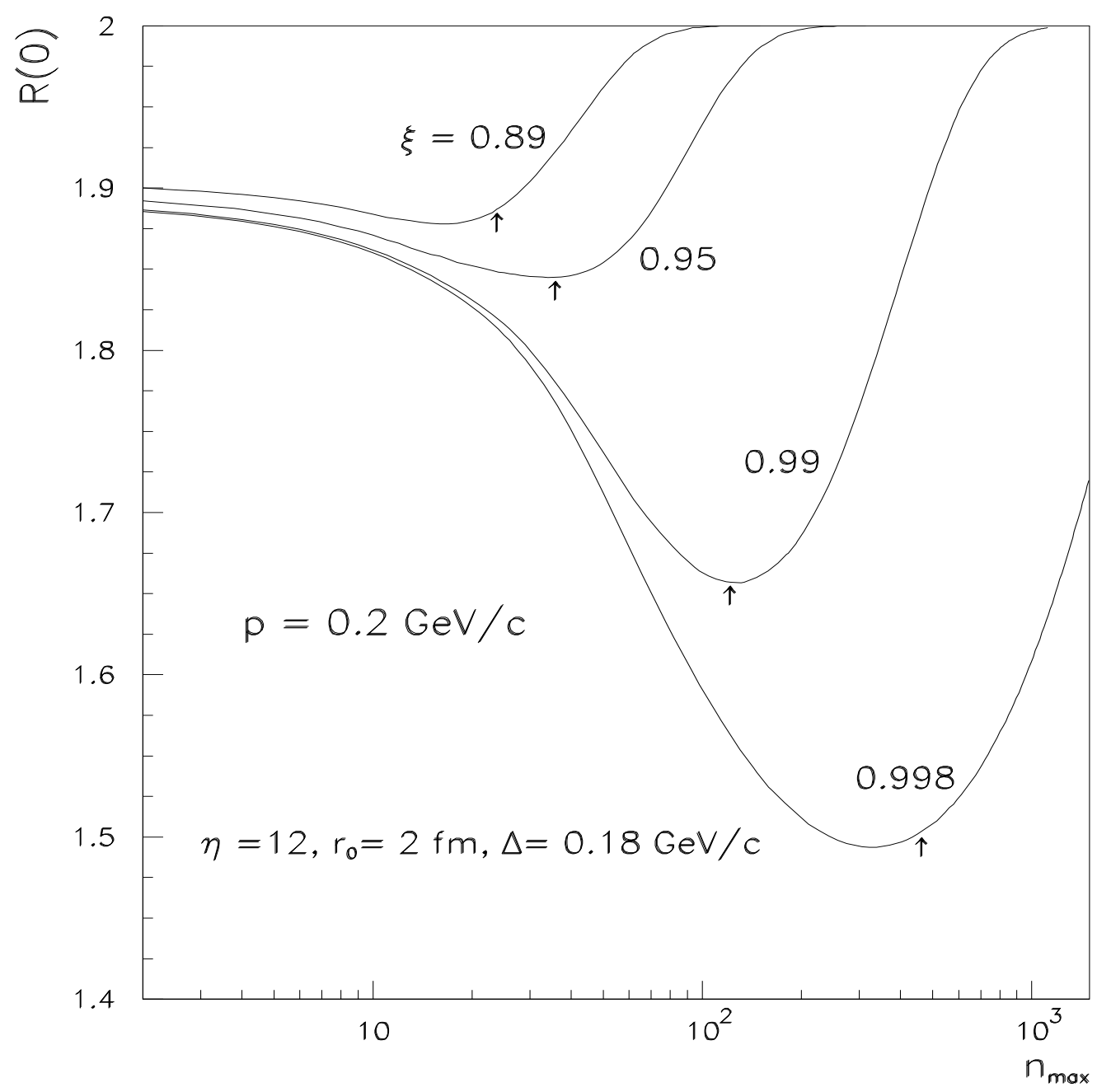

FIG. 10. The intercepts of the semi-inclusive correlation function including the pion multiplicities from 0 to $n_{\text {max }}$ as functions of $n_{\max }$ for different values of the density parameter $\xi=0.89,0.95,0.99$ and 0.998 ; the arrows indicate the corresponding mean multiplicities $\langle n\rangle=22.3,33.5,113.7$ and 433.8. The conditions are the same as in Fig. 8. 